\title{
1 Structural brain connectivity predicts acute pain after mild traumatic
}

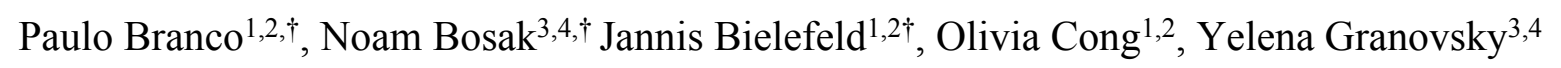

Itamar Kahn ${ }^{5}$, David Yarnitsky ${ }^{3,4, \&}$, A. Vania Apkarian ${ }^{1,2, \& *}$

Author affiliations:

7 'Department of Neuroscience, Northwestern University Feinberg School of Medicine, Chicago, 8 USA

$9{ }^{2}$ Center for Translational Pain Research, Northwestern University Feinberg School of Medicine,

10 Chicago, USA

11 Rappaport Faculty of Medicine, Technion - Israel Institute of Technology, Haifa, Israel

$12{ }^{4}$ Department of Neurology, Rambam Health Care Campus, Haifa, Israel

13 Department of Neuroscience and Zuckerman Mind Brain Behavior Institute, Columbia

14 University, New York, USA.

16 *Correspondence to: Dr. A. Vania Apkarian (AVA)

17 a-apkarian@northwestern.edu

19 These authors contributed equally to this work.

$20 \&$ These authors also contributed equally to this work.

23 Short title: Brain connectivity predicts pain in mTBI 


\section{Abstract}

25 Mild traumatic brain injury, mTBI, is a leading cause of disability worldwide, with acute pain

26 manifesting as one of its most debilitating symptoms. Understanding acute post-injury pain is

27 important since it is a strong predictor of long-term outcomes. In this study, we imaged the brains

28 of 172 patients with mTBI, following a motorized vehicle collision and used a machine learning

29 approach to extract white matter structural and resting state fMRI functional connectivity measures

30 to predict acute pain. Stronger white matter tracts within the sensorimotor, thalamic-cortical, and

31 default-mode systems predicted $20 \%$ of the variance in pain severity within 72 hours of the injury.

32 This result generalized in two independent groups: 39 mTBI patients and 13 mTBI patients without

33 whiplash symptoms. White matter measures collected at 6-months after the collision still predicted

34 mTBI pain at that timepoint $(\mathrm{n}=36)$. These white-matter connections were associated with two

35 nociceptive psychophysical outcomes tested at a remote body site - namely conditioned pain

36 modulation and magnitude of suprathreshold pain-, and with pain sensitivity questionnaire scores.

37 Our validated findings demonstrate a stable white-matter network, the properties of which

38 determine a significant amount of pain experienced after acute injury, pinpointing a circuitry

39 engaged in the transformation and amplification of nociceptive inputs to pain perception. 


\section{Introduction}

41 Traumatic brain injury is a leading cause of death and disability in the United States(1), with

42 important socioeconomic costs. Motor vehicle collisions are a frequent cause of mild TBI (mTBI),

43 which often is accompanied with whiplash associated disorders (WAD) given the quick shift of

44 forces during a crash and the consequent abrupt and brisk back-and-forth movement of the neck.

45 These patients usually present with a range of clinical symptoms including stiffness, dizziness, nausea, and mental confusion, but the most frequent and debilitating clinical manifestation is pain

47 in the head/neck area(2), which may last from days to weeks and - for a large proportion of patients

48 - can become chronic(3). Managing pain post-injury is of utmost importance since it reflects on

49 the patients' recovery process: mTBI patients with acute pain-related disabilities are at higher risk

50 for chronic pain(3) and are more likely to develop clinically significant anxiety, depression, sleep

51 disturbances, and post-traumatic stress disorder(4)

Early acute pain after mTBI is, however, poorly understood. The etiology of pain does not

53 map well onto injury-related imaging findings(5),(6),(7) and is only partially explained by

54 psychological and psychophysical pain characteristics $(8,9)$. It is known that psychological factors

55 play a role in early acute pain, with state anxiety, depression, and pain catastrophizing modestly

56 explaining additional variance in pain severity(10). At the chronic stage, it is also known that these

57 patients have altered psychophysical pain indices, including hyperalgesia to painful stimuli,

58 inefficient conditioned pain modulation, and enhanced temporal summation of pain(11). While it

59 is thought that both pro-nociceptive and affective/emotional processes are driven by brain-centric

60 mechanisms, so far, no studies have examined the brains of these patients in relation to acute mTBI

61 pain. Consequently, how the central nervous system reacts to, is changed by, or can predispose

62 someone to experience pain after acute mTBI injury is unknown. Peering into the subject's brains 
63 and exploring the mechanisms underlying pain at the early acute stage is a promising approach to

64 resolve the relative contribution of psychological, psychophysical, and nociceptive processes in

65 post-injury pain and can shed light on the mechanisms that underlie pain perception immediately

66 after an emotionally charged, pain-inducing incident.

In this study, we used MRI to study brain functional and structural properties of mTBI

68 patients after a motorized vehicle accident and probed for properties that can determine or

69 predispose patients to experience pain after injury. Using a machine-learning approach, we

70 examined the functional and structural brain networks associated with early, acute pain. This study

71 sheds light on the properties and emergence of pain, particularly after injury, with implications for

72 the treatment and management of mTBI.

\section{Results}

\section{Predicting pain after mTBI using functional and structural connectivity}

76 We used a machine-learning approach to predict pain from resting-state functional magnetic

77 resonance imaging (rsfMRI) and diffusion tensor imaging (DTI) brain connectivity. Discovery

$78\left(70 \%\right.$ of the data, $\mathrm{N}_{\text {rsfMRI }}=94$ and $\mathrm{N}_{\mathrm{DTI}}=88$ after outlier exclusion $)$ and hold-out $\left(\mathrm{N}_{\mathrm{rsfMRI}}=43\right.$

79 and $\mathrm{N}_{\mathrm{DTI}}=37$ after outlier exclusion) datasets were used to build the model and assess

80 generalizability (see Fig. 1 and methods for an explanation of the machine-learning pipeline).

81 For structural connectivity, after 10-folds cross-validation (CV), the best $p$ threshold for

82 univariate feature selection was determined to be $p=0.01$ (negative features model, highest CV

$83 r_{\text {predvsactual }}=.33$; positive model n.s.), leading to the selection of 26.5 features on average, which

84 mostly appeared inconsistently across CV folds (see Supplementary Fig. 1). 
Only three white-matter (WM) tracts were selected on all cross-validation folds: tract 1 ,

86 left Precentral gyrus - left Postcentral Gyrus; tract 2, left Thalamus - left Superior Parietal Lobule;

87 and tract 3, right Planum Polare - left Superior Lateral Occipital Cortex (Fig. 2A). Within the

88 discovery dataset, these three features, together, show an $r_{\text {predvsactual }}=0.47$ [95\% CI: $0.324,0.572$ ],

89 and $p<.001$ (Fig. 2B). By applying the linear model built on the discovery dataset, we were able

90 to predict patient's pain in the hold-out dataset (Fig. 2B): the model showed a $r_{\text {predvsactual }}=0.43$

$91 \quad[95 \%$ CI: 0.158, 0.593], $p=.007$, thus suggesting generalizability. We further examined if the WM

92 connectivity model could predict pain in the sub-sample of mTBI patients without whiplash

93 symptoms (WAD score $=0, \mathrm{~N}=13$ ). Since these subjects were not used in previous analyses, they

94 represent a second independent group on which generalizability can be assessed. The model was

95 again successful at predicting patients' pain and had a $r_{\text {predvsactual }}=.53[95 \% \mathrm{CI}=0.058,0.697]$,

96 see Fig. 2B, although this correlation did not reach significance $(p=.061)$, potentially due to the

97 small sample size. Meta-analytical prediction metrics were obtained by assessing the pooled

98 prediction performance in the three datasets $(\mathrm{N}=138)$. Results replicate the previous findings,

99 with a $r_{\text {predvsactual }}=.44[95 \% \mathrm{CI}=0.320,0.532], p<.001$, see Fig. 2C. For the three tracts, WM

100 connectivity was significantly correlated with pain ratings (all $r \mathrm{~s}>-0.3, p \mathrm{~s}<.001$ ), with higher

101 connectivity strengths leading to lower observed pain (Fig 2D). Finally, to discard the possibility

102 that the WM connectivity strength is associated with the severity of the clinical manifestation we

103 explicitly compared the connectivity strength of the three tracts between patients with a WAD

104 score of 0, 1 and 2, reflecting whiplash signs and symptoms severity. A one-way ANOVA showed

105 no significant differences in connectivity between groups $(F(2,135)=1.11, p=.33$, Fig. $2 \mathrm{E})$. 
using this approach, rsfMRI is unable to predict early acute pain. Further analyses are reported for

109 structural analyses only.

\section{Model predictions do not depend on age, sex, total intracranial volume}

112 We next asked whether this finding could be explained by confounds, such as age, sex, and total

113 intracranial volume (TIV). We conducted a sequential linear regression on the discovery dataset

114 with age, sex, and TIV as independent variables and pain as the dependent variable. This model

115 was not significant $(p=.074)$ and only explained $4.6 \%$ of the variance in pain ratings. Adding the

116 structural connectivity score to this model resulted in a significant model that explained $22 \%$ of

117 the variance in the pain ratings $(p<.001)$; this increase in explained variance was statistically

118 significant (model 1 vs. model $2, F[1,83]=20.29, p<.001)$.

\section{Spatial specificity within thalamic and somatosensory networks}

121 Given that the sensorimotor network follows a somatotopic organization and the thalamus has

122 well-characterized nuclei implicated in multiple sensory and affective processes, we further tested

123 the spatial specificity of both regions. Thus, brain regions from the left precentral and postcentral

124 gyrus were parcellated based on their body-part representation (face, arm, trunk, leg) using masks

125 published previously(12) (Fig. 3A). The connectivity between each pair of regions was estimated

126 and correlated with baseline pain. The number of connections between the precentral-postcentral

127 face $(r=-.26, p=.004$, Fig. 3A), the precentral-postcentral arm area $(r=-.24 p=.008)$, and

128 precentral arm and postcentral face $(\mathrm{r}=-.21, p=.019)$, were significantly associated with pain

129 ratings. 
We also examined the left thalamus using the FSL thalamic connectivity atlas(13), containing seven regions of interest (ROIs), each highlighting connectivity to different brain areas

132 (prefrontal, premotor, motor, sensory, parietal, occipital, temporal). The connectivity between each

133 thalamic ROI and the left Superior Parietal Lobule (SPL) was calculated and correlated with

134 reported pain. We found evidence of spatial specificity - the thalamic nucleus most predictive of

135 pain was the subregion mostly connected to the Parietal cortex, corresponding spatially to the 136 posterior lateral thalamus area $(r=-.30, p<.001$, Fig. 3B); further, we also observed strong 137 associations between the connectivity of the prefrontal ROI and left SPL, tapping into more 138 anterior-medial thalamus $(r=-.30, p<.001)$. We further observed significant correlations between 139 lateral thalamic nuclei connecting to sensory $(p=.009)$, motor, and premotor areas $(p=0.027$, and $140 p=.017$, respectively). The other thalamic ROIs (connected to the temporal and occipital cortex) 141 were not significantly associated with pain.

\section{Predictive brain parameters are associated with psychophysical indices of pain}

\section{4 sensitivity}

145 To ground these findings in clinical properties, we tested for an association between brain,

146 psychophysical and clinical parameters. We found significant positive associations between WM

147 connectivity strength of all tracts and the conditioned pain modulation (CPM, $r=.21, p=.019)$,

148 the temperature at which subjects rated a pain of 50/100 (suprathreshold pain, termed Pain50(8),

$149 r=.20, p=.027)$, as well as negative associations with scores in the pain sensitivity questionnaire

150 (PSQ, $r=-.19, p=.038$ ). Individually, pressure-pain CPM response was mostly associated with

151 precentral-postcentral connectivity $(r=.25, p=.006)$, while connectivity between the right Planum

152 Polare and the superior Lateral Occipital Cortex was associated with the perceived stress scale $(r$ 
$153=.20, p=.034)$. Other clinical and psychological parameters did not show significant results (Fig.

154 4A).

155 Given that these clinical parameters have been previously associated with pain ratings in

156 these individuals $(8,9)$, we further wanted to disentangle the relative contribution of these

157 psychological and psychophysical dimensions to that of the brain. To do so, we performed a

158 relative importance analysis (using the $\mathrm{R}$ package relaimpo(14)). While the total model with the

159 four parameters (CPM, Pain50, PSQ, and Brain Connectivity) explained $33.6 \%$ of the variance in

160 pain ratings (Fig. 4B), brain connectivity alone explained 15\% unique variance in pain ratings.

161

162 The predictive ability of the model is not contingent on machine-learning

163 pipeline

164 In order to ensure these results were not contingent on the modelling approach, we repeated the

165 analyses using a simple linear regression combining both positively and negatively associated

166 tracts and without summing the tracts into a single value. After cross-validation, the best

167 performing threshold for feature selection was $p=.005\left(\mathrm{CV} r_{\text {predvsactual }}=.15\right)$, leading to two highly

168 reliable features (tract 1, left Precentral gyrus - left Postcentral Gyrus; tract 2, left Thalamus - left

169 Superior Parietal Lobule; tracts 1 and 2 from the previous analyses). Within the discovery dataset,

170 the model obtained an $r_{\text {predvsactual }}=0.37$ [95\% CI: $\left.0.192,0.503\right], p<.001$. Within the hold-out

171 dataset, this model obtained a $r_{\text {predvsactual }}=.40[95 \% \mathrm{CI}: 0.115,0.577], p=0.014$. Unsurprisingly,

172 predictions and statistical results from machine-learning and linear regression models were similar,

173 with the correlation of predicted pain values from both methods being $r=.90$ and .96 for the

174 discovery and hold-out datasets, respectively. 
176 Structural connectivity parameters are stable over time, and explain future

\section{7 pain}

178 Since this study is part of a larger project aimed at discovering brain predictors of transition into

179 chronic pain in mTBI patients, we examined the pain-ratings of these patients over time. The

180 strength of WM connectivity at baseline was associated with patients' pain one, three, and six

181 months after baseline (all $p s<.05$, Fig. 4E). This effect, however, was substantially reduced when

182 controlling for pain ratings at baseline. We then assessed whether these connectivity indices were

183 static in time; hence, we examined DTI data from a sub-sample of these patients that repeated the

184 MRI protocol at six months $(\mathrm{N}=36)$ and one year $(\mathrm{N}=13)$ after injury. We compared, within-

185 subject, whether the connectivity strength of the three tracts changed over time. Paired t-tests show

186 that the connectivity does not change over time $(p=.74, p=.52,6$ months and 1 year, respectively,

187 Fig.5A). Importantly, DTI parameters collected at six months post-injury were also significantly

188 associated with pain at six months $(r=-41, p=.012$, see Fig. 5B). This result, however, did not

189 hold at one year after injury $(r=.25, p=.4)$, despite DTI parameters not changing and a significant

190 proportion of patients (50\%) still reporting pain.

192 Discussion

193 In this study, we examined the brains of mTBI patients suffering from early acute pain, attempting

194 to characterize brain networks underpinning acute pain after a motorized vehicle collision injury.

195 Our results can be summarized in four key points. First, we show that white-matter brain properties

196 - but not functional properties - explain a sizable variance of the pain after mTBI, hence

197 predisposing patients to report more pain after acute injury. Second, we demonstrate that these

198 findings are not dependent on injury-related, clinical, or demographic characteristics of the 
199 patients. Third, these white-matter connections map well onto physiological-psychological

200 characteristics, namely through interactions with quantitative sensory testing parameters and pain

201 sensitivity; together, these parameters can explain a third of the pain variability irrespective of

202 tissue damage. Fourth, these connectivity parameters do not change up to a year after the injury,

203 and connectivity metrics collected at baseline, as well as the same parameters collected at six

204 months, are able to predict subjects' pain at six months after the initial injury. Together, these

205 findings suggest an a-priori predisposition to pain grounded in brain WM properties, which results

206 in higher pain ratings after acute injury. These findings shed light on brain mechanisms underlying

207 pain sensitivity and inform the current literature of pain perception of a poorly understood patient

208 population - mTBI patients.

Our study shows that an important part of the reported post-injury pain can be accounted

210 for by brain imaging features alone. Although previous studies have successfully predicted

211 experimental pain(15) and tonic pain(16) in healthy participants, as well as pain in patients at the

212 chronic stage(16), this is, to the best of our knowledge, the first demonstration that early acute

213 pain, isolated from plastic changes that occur over time after an injury, can be predicted based on

214 brain structure. The strength of the evidence here lies in the clinical sample studied: these patients

215 did not have any pain before injury and were scanned within hours of the accident, making them

216 an ideal group to study injury-related, early acute pain in an ecological manner. The influence of

217 the brain in pain perception is clear in the literature, but reports using brain structure and function

218 to predict pain, particularly post-injury acute pain are scarce. Spisak and colleagues(17) have, for

219 instance, recently showed that brain functional connectivity can predict pain sensitivity in healthy

220 participants, which points to a predisposition to pain grounded on their underlying

221 connectome(17). Here, we extend that idea by showing that injury-related pain can also be 
222 predicted from the structural connectome. Within mTBI/whiplash literature, there is an ongoing

223 discussion about the origin of the reported pain: the majority of the time, it is difficult to find

224 imaging correlates of structural damage(5-7), and the patient's pain is affected by psychological

225 aspects, such as psychological distress, anxiety, depression(8,18), and seem related to insurance

226 pay-off and work-related disabilities(19). Mapping post-injury pain to brain measures of

227 diffusivity solidifies, at least in part, the organic basis of the patients' pain. The fact that the

228 networks subtending this prediction are classically implicated in nociception (rather than more

229 limbic brain regions implicated in negative affect), together with associations between these

230 networks and psychophysical/psychological indices related to pain sensitivity, in particular,

231 conditioned pain modulation, and pain sensitivity questionnaire scores, further adds that at this

232 early time-point, pain sensitivity profiles-but not psychological aspects-dominate the

233 association with pain ratings, which fits well with previous clinical findings(8).

234 Three WM connections reliably predicted pain in these patients: they appeared on all cross-

235 validated folds, predicted pain within-sample and predicted pain in two additional independent

236 groups of patients. The first, and strongest, predictive feature of post-injury pain was the number

237 of WM connections between the primary motor and sensory regions of the brain. The implication

238 of these two regions in pain perception is not novel, given that they are well-known components

239 of nociceptive pathways(20). It is also well established in animal studies that the precentral and

240 postcentral gyrus have reciprocal structural connections, $(21,22)$ which are crucial for sensorimotor

241 integration(23-28). In humans, imaging data supports the idea that there is strong information

242 sharing between these two regions, both during task ${ }^{30}$ and during rest(30), which has been

243 theorized to have a role in pain perception(31). The strength of connectivity in this area was also

244 associated with conditioned pain modulation and pain sensitivity questionnaire scores, further 
245 suggesting that sensorimotor connectivity is related to pain sensitivity. This is also coherent with

246 previous work showing that higher cortical density in the somatosensory cortex leads to less

247 experienced pain while performing quantitative sensory tasks in healthy subjects(32) and that

248 denser cortical thickness of the somatosensory cortex is associated with higher pain thresholds(33).

249 In fact, it is known that the sensorimotor area is able to modulate pain, namely through direct

250 projections to the thalamus(34). Our findings also show spatial specificity that coincide with the

251 site of injury: connectivity between precentral and postcentral face and arm area (corresponding

252 to the head/neck representation in the motor homunculus), as well as connectivity between the

253 precentral face and postcentral trunk area (head/neck area in the sensory homunculus) are mostly

254 driving these results.

255 Similarly, there is ample evidence supporting the association between the thalamus and

256 pain as this region is the termination site of the spinothalamic tract and of nociceptive information

257 relay to the cortex. The SPL is a multisensory, high-level integrative site for pain(35) and has also

258 been associated with top-down modulation of pain(36). Connections between the thalamus and

259 SPL have been described in tracing studies in rhesus monkeys, showing that the SPL projects to

260 the ventrolateral (VL) and posterior lateral (VPL) thalamic nuclei(37). VL regions of the thalami

261 are also connected to the parietal cortex in humans(13). This is precisely the thalamus region best

262 predicting pain, as seen in the thalamic parcellation analysis. One recent study has shown that

263 connections between the VL/VPL and the SPL (and the postcentral gyrus) are associated with the

264 pathophysiology of pain(38), and higher gray matter density in the SPL has also been linked with

265 pain sensitivity(32). Considering that SPL acts as a top-down inhibitor of sensory stimuli and given

266 that thalamic-cortical projections from VPL to SPL are central to chronic pain, it is tempting to 
267 suggest that this connectivity may play a role in the inhibition (or exacerbation) of nociceptive 268 signals arriving at the thalamus.

269 The third tract engaged in mTBI pain connects the planum polare to the superior lateral 270 occipital cortex. The superior lateral occipital cortex (which in the Harvard-Oxford cortical atlas

271 extends into the IPL, see Fig. 2C) is a main integral part of the default-mode network with ample

272 evidence of manifesting in sensory and pain-related behavior(39). Its connection with the planum

273 polare is surprising, as this region is traditionally involved in auditory processing(40). One critical

274 aspect is that this region is located within an area rich in crossing-fibers (within the external

275 capsule) and near important pain-related regions, such as the insula, the secondary somatosensory

276 cortex, and the posterior operculum. Therefore, it is likely that the loss of clear directionality within

277 the gray matter (a well-known limitation of DTI $(41,42)$ ) led to the fibers tractography algorithm

278 to terminate prior to reaching the actual target. This explanation is speculative and requires future 279 studies.

280 An important question to discuss is whether these brain features reflect injury-related

281 parameters, short-term plastic changes, or rather an a-priori predisposition to pain hard-wired in

282 the brain. Our results favor the latter. Considering an injury-related explanation to these results,

283 one could argue these parameters are mapping tissue injury as a proxy for pain: it is known that

284 whiplash-like centrifugal forces may cause axonal micro-lesions that are not detectable in 285 conventional scanning protocols(43); and these brain lesions could, in turn, reflect how severe the $286 \mathrm{mTBI} /$ whiplash injury is(44), leading to higher reported pain. We find this possibility unlikely:

287 first, we excluded patients with WAD scores above three and patients with obvious signs of brain 288 damage. Second, we did not find associations between connectivity strength and whiplash severity, 289 and the model was able to predict pain in the group of patients with no whiplash-like symptoms. 
290 Lastly, there are accounts that white-matter properties, namely fractional anisotropy and median

291 diffusivity, are unaffected following $\operatorname{mTBI}(45)$. Another possibility is that this connectivity is

292 reflecting short-term plastic changes caused by the new pain state. Our data does not support this

293 hypothesis either: participants were scanned within $72 \mathrm{~h}$ of the injury. It is unlikely that white-

294 matter properties changed over a timespan of hours and ceased to change any further over longer

295 timespans. There is evidence of short-term white-matter diffusivity changes in other

296 contexts(46,47), but if the system is so malleable, one would expect to observe diffusivity changes

297 over longer times too, especially as many participants gradually recover from their acute pain. We

298 in fact observe no changes in connectivity strength in these WM networks from the time of the

299 accident to six months and up to a year. Moreover, these same connections measured at six months

300 post-injury also predict the reported pain at the same point in time, further adding strength to the

301 within-subject reliability of this model. In conclusion, we consider it quite unlikely that our results

302 reflect injury-related parameters or plastic changes that occur in the timespan of the data-

303 collection; our data instead favors the idea that observed WM properties reflect an a priori brain

304 predisposition for pain sensitivity.

305 While white-matter properties predicted pain quite successfully, fMRI functional

306 connectivity did not. It is thought that functional connectivity is primarily supported by structural

307 connectivity(48), and given the success of using resting-state to study pain sensitivity(17) and

308 phasic pain(16), it is somewhat surprising that functional connectivity was not informative of acute

309 mTBI pain. It is, however, important to point out key differences between DTI and rsfMRI

310 techniques. While white-matter properties are relatively stable and should reflect primarily long-

311 term changes in brain structure (i.e., more trait-like), rsfMRI should better reflect the emergence

312 of a state within a given macro-structure (i.e., more state-like(49)). Given that patients were 
313 scanned within $72 \mathrm{~h}$ of a motorized vehicle accident, an often emotionally charged event that will

314 no doubt imprint a state of anxiety and distress on the patient, it may be difficult to identify a

315 reliable and solid pattern that generalizes across subjects, particularly given the heterogeneity of

316 the psychological factors and their influence on functional connectivity. Importantly, the fact that

317 only white matter is predictive of pain post-injury in the patients should be construed as further

318 evidence that they represent stable brain circuitry implicated in the transformation and

319 amplification of nociceptive inputs, rather than simply capturing the perception (state) of pain per

320 se.

321 This study has some limitations. We lack a group of healthy participants, which could be

322 used to directly ascertain if the connectivity profiles are affected by the injury itself. Also, it is

323 necessary to mention that the absolute agreement between predicted pain and observed pain is

324 subpar (i.e. participants with 0 pain are predicted to have an average of 30 pain). Nonetheless, the

325 goal of this study was to infer brain mechanisms from early acute pain, rather than minimizing the

326 error in out-of-sample prediction. Finally, our sample is quite heterogenous in several respects,

327 including acute treatment, type of car accident, and perhaps others. Naturally, there is an vast

328 number confounds that could have been controlled for to improve the predictive ability of the

329 model; here, we favored a less constrained approach as it provides strength to the predictive power

330 of our analyses - it predicts pain regardless of uncontrolled confounds.

331 In conclusion, this study shows that measures of brain diffusivity in white-matter tracts

332 predict an important part of acute pain shortly after an injury. These tracts are implicated on

333 nociceptive and pain-related circuitry, which may reflect preexisting pro-nociceptive or anti-

334 nociceptive influences that either amplify or diminish the cortical interpretation of the injury-

335 related nociceptive barrage into the subjectivity of pain. These findings lead to new questions for 
336 future research: are these structural networks indeed generalizable across pain conditions? Can

337 these connections predict acute, experimental pain in healthy subjects? The study of white-matter

338 diffusivity in early acute pain thus opens new avenues for studying pain sensitivity and establishes

339 dependence of pain perception, in part, on hard-wired central nervous system structures. It further

340 provides support for the organic basis of pain perception in mTBI-whiplash patients, which is often

341 labeled as purely psychogenic in nature.

\section{Materials and methods}

\section{Participants}

345 Participants included in this study were recruited at the Rambam Health Care Campus emergency

346 department after suffering an mTBI injury from a motor vehicle accident at a maximum of 24

347 hours before their visit. They signed an informed consent in agreement with the Declaration of

348 Helsinki. The study was approved by the institutional review board of Rambam Health Care

349 Campus (ref. 0601-14). All patients had direct or indirect head and neck injury, a Glasgow Coma

350 Scale score of 13 to 15 and no subsequent decline, and were over 18 years of age. We excluded

351 patients with imaging-based traumatic brain findings and more than 30 minutes of

352 unconsciousness. Patients were also excluded if they had other major bodily injuries, prior chronic

$353 \mathrm{head} /$ neck pain, injuries in the head/neck area within one year prior to the current injury, and

354 neurological diseases compromising the interpretation of brain function (e.g., neurogenerative

355 diseases).

356 We recruited 249 patients, out of which we excluded 17 patients who did not fulfill mTBI

357 clinical criteria, 54 patients who did not undergo MRI (claustrophobia, or not willing to 358 participate), four patients who did not report baseline pain, and two patients with incidental MRI 
findings. Given the current discussion on the common etiology and symptomatology of patients with WAD and mTBI(50), we decided to homogenize our sample by studying only patients

361 fulfilling both WAD and mTBI diagnosis (90\% of patients). Hence, 15 patients showed no signs

362 of WAD (WAD score $=0$ ) and were excluded from the main analyses. They were, however, used

363 post-hoc, as an additional validation group. The remaining 157 subjects (mean age $=37.3 \pm 12$

364 years, 86 male, average pain $=56.6 \pm 26.2$, range $0-100)$ were analyzed. Not all subjects completed 365 all MRI protocols (detailed exclusions shown in Supplementary Fig. 1).

\section{Study design and procedures}

368 After the initial emergency room visit, patients were informed about the study and were consented.

369 Patients were then scheduled for a visit within 3 days of the injury (mean 1.7 days, range $=0-3$ ),

370 where they performed an MRI scan (structural, resting-state, and diffusion MRI protocols).

371 Participants were asked to refrain from taking analgesic medication for 24 hours before the data

372 collection. During the visit, patients were asked to rate their average pain over the previous 24

373 hours, separately, for their neck and head using a numeric rating scale (NRS, 0-100; 0: no pain,

374 100: worst imaginable pain); their current pain was determined to be the highest of the two.

375 Patients also performed a series of clinical, psychological, and psychophysical tests, which are

376 explained in detail elsewhere(8). Briefly, they filled a battery of psychological questionnaires

377 measuring anxiety and depression $(\operatorname{HADS}(51))$, pain catastrophizing (PCS(52)), pain sensitivity

378 (PSQ(53)), stress (PSS(54)), and underwent quantitative sensory testing (conditioned pain

379 modulation with heat and pressure stimuli, and temporal summation protocols with pressure and 380 electrical stimuli). Some of these data are featured in other publications, examining psychophysical 381 and psychological predictors of early acute mTBI pain $(8,9)$. 


\section{Scanner parameters}

384 All images were collected on a 3T (MR 750, SIGNA 20, GE Medical Systems, Milwaukee, USA) 385 scanner with a 16-channel head-coil. High-resolution T1-weighted images were collected with the 386 following parameters: field of view $(\mathrm{FOV})=256 \times 256 \mathrm{~mm}^{2}$, flip angle $=12^{\circ}$, slice thickness $=1$

$387 \mathrm{~mm}$, in-plane pixel size $1 \times 1 \mathrm{~mm}^{2}$, axial slices $=172$. Diffusion weighted images were collected, 388 with the following parameters: $\mathrm{TR}=10000 \mathrm{~ms}, \mathrm{TE}=82$, FOV $=256 \times 256 \mathrm{~mm}^{2}$, slice thickness $389=2 \mathrm{~mm}$, in-plane pixel size $1 \times 1 \mathrm{~mm}^{2}$, axial slices $=68$, and number of directions $=60$ with a b390 value of $1000 \mathrm{~s} / \mathrm{mm}^{2}$. Five volumes with no diffusion weighting (b-value $=0 \mathrm{~s} / \mathrm{mm}^{2}$ ) were 391 acquired at the start of the protocol. Resting-state fMRI images were collected with an EPI 392 sequence with the following parameters: TR $=2000 \mathrm{~ms} ; \mathrm{TE}=30 \mathrm{~s} ; \mathrm{FOV}=220 \times 220 \mathrm{~mm}^{2}$; flip 393 angle $=75^{\circ}$; slice thickness $=3.4 \mathrm{~mm}$; in-plane pixel size $3.44 \times 3.44 \mathrm{~mm}^{2}$ and axial slices $=43$.

394 The first $8 \mathrm{~s}$ of acquisition in each run were excluded due to T1 equilibration effects. Participants 395 were instructed to stay as still as possible, close their eyes, not fall asleep, and think of nothing in 396 particular.

\section{Data preprocessing}

399 All images were preprocessed using tools from the FMRIB FSL library (FSL 6.0.4(55)). Structural 400 images were skull-stripped using $\operatorname{BET}(56)$ and segmented into white-matter, gray-matter, and 401 cerebrospinal fluid (CSF) masks using FAST(57). As a proxy of total intracranial volume (TIV), 402 the volume of grey-matter and white-matter masks were summed.

403 For resting-state fMRI images, data were skull-stripped, slice-time corrected, smoothed 404 using a $6 \mathrm{~mm}$ FWHM kernel, and filtered using a band-pass filter (.02 to .001 Hz). To further 
remove signal of no interest, we used a strict denoising procedure: the six head motion parameters, their squared parameters, and the temporal derivatives of both were estimated. Signal from the WM and CSF was identified by averaging the time course within the respective masks; these masks were eroded once to ensure no overlap between tissue types. The 24 movement parameters, together with the WM and CSF time courses, were then regressed out from the data through

410 multiple regression. Finally, high motion timepoints and their immediately adjacent volumes were

411 removed (motion scrubbing) if they exceeded one of three criteria: timepoints with a framewise

412 displacement larger than 0.7, timepoints exceeding 2.3 standard deviations of the mean signal, or

413 a derivative of the root mean squared over voxels (i.e. DVARS) larger than 2.3. Functional images

414 were normalized to standard MNI space using a two-step process. First, they were registered from

415 functional to $\mathrm{T} 1$ structural space using boundary-based registration. Then, images were

416 transformed from structural space to MNI by applying a 12 degrees-of-freedom linear

417 transformation (FLIRT(58)), followed by a non-linear transformation (FNIRT). Subjects with less

418 than 5 minutes of resting-state data after scrubbing were excluded $(\mathrm{N}=3)$. Four additional subjects

419 were excluded from analyses due to technical/image problems (see Supplementary Fig. 1). The

420 final rsfMRI sample included 141 subjects.

Diffusion weighted images were visually inspected for obvious artefacts and corrected for

422 eddy current distortions and head movement using EDDY(59). Then, a tensor was fit to the data,

423 and principal directions were calculated using DTIFIT. To account for possible crossing-fibers, a

424 Bayesian estimation of the principal directions was performed with BEDPOSTX(60), modelling

425 up to three principal directions. Transformation matrices from subject space to MNI were obtained

426 using FLIRT and FNIRT. For diffusion tensor imaging quality control, we used QUAD and

427 SQUAD(61); subjects scoring as group outliers in absolute/relative displacement $(\mathrm{N}=5)$ or signal 
428 to noise ratio/contrast to noise ratio $(\mathrm{N}=1)$ were excluded. Eight other subjects were excluded due

429 to technical issues with the data (see Supplementary Fig. 1). The final DTI sample includes 129

430 subjects.

\section{Extraction of brain connectivity features}

433 To sample brain connectivity at a comprehensive yet interpretable scale, we used the Harvard-

434 Oxford Cortical and Subcortical atlas. This atlas is composed of 115 brain regions and spans major

435 brain networks. Due to field-of-view coverage and in line with our previous research, we excluded

436 regions of interest (ROIs) within the cerebellum and the brainstem, resulting in a total of 105

437 cortical and subcortical ROIs (Supplementary Fig. 2A, B). For functional connectivity analyses,

438 the average time course of each ROI was correlated to the time courses of all other ROIs, resulting

439 in a $105 \times 105$ connectivity matrix. Since the matrix is symmetric, only the lower triangle of the

440 connectivity matrix was kept, for a total of 5460 features per subject. Correlation $(r)$ values were

441 converted to $\mathrm{z}$ scores using the Fisher r-to-z conversion to approximate a normal distribution. For

442 structural connectivity analyses, we first generated a white-matter/gray-matter interface mask by

443 intersecting FSL's white-matter and gray matter tissue priors at 25\% probability (Supplementary

444 Fig. 2C). Then, probabilistic tractography was ran using probtrackx2(60), seeding only from

445 voxels within the ROIs that are included in the WM/GM interface mask. For each voxel within

446 each ROI, 5000 streamlines were seeded, and the number of connections reaching each other ROI

447 was counted, again resulting in a $105 \times 105 \mathrm{WM}$ connectivity matrix per subject. As distance

448 between ROIs can influence connectivity results and introduce a head-size bias, WM connectivity

449 count was corrected for distance by multiplying the number of streamlines by the Euclidian

450 distance between the two ROIs (--pd option in probtrackx2). Finally, since the connectivity matrix 
451 was undirected, we averaged the upper and lower triangles of the matrix, to obtain a single ROI-

452 to-ROI value indicative of probability of connectivity. As with rsfMRI connectivity, this resulted

453 in 5460 structural connectivity features.

\section{Machine-learning approach}

456 We built a machine-learning model to predict the patients' reported pain, using whole-brain,

457 structural and functional connectivity between 105 ROIs, yielding 5460 independent variables per

458 method. To simplify this overdetermined fit - we studied 141 and 129 participants for rsfMRI and

459 DTI, respectively - we used a well-established method, Connectome-based Predictive Modelling,

$460 \mathrm{CPM}(62,63)$. This method simplifies the fit by reducing the number of features through a

461 univariate feature selection, followed by a simplified form of coefficient regularization (see

462 below). Univariate feature selection is performed by correlating all 5460 brain features against a

463 dependent variable (Fig. 1A); these are then split into two sets of features, positively and negatively

464 correlated to the dependent variable, which yield two separate models. Features correlating

465 significantly below a given p-value are selected, which are summed to obtain one single parameter

466 that characterizes the connectivity strength of a given set of tracts (Fig. 1B). Finally, the model is

467 built by fitting a line between the dependent (pain) and independent variable (brain connectivity,

468 Fig. 1C). Out of the 5460 features, this method reduces the fit to two coefficients (slope and

469 intercept) per (positive and negative) model, which can then be tested for generalizability in the

470 hold-out sample.

471 To enable model generalizability, we first split the dataset into two: a discovery dataset

472 ( $70 \%$ of the data) on which the model was built and a hold-out dataset ( $30 \%$ of the data) on which

473 the model was validated. This was performed using the Kennard-Stone algorithm(64), which 
474 divides a dataset into two or more partitions while matching them for given parameters, which we

475 conducted based on age, sex, and TIV. Further, within the discovery dataset, we also performed a

476 10-fold cross-validation approach (Fig. 1D). The latter was needed for two goals: 1. To impartially

477 determine the (otherwise arbitrary) univariate feature selection p-threshold; 2. To minimize model

478 overfitting (more details in Supplementary Material). Thus, we divided the discovery dataset into

47910 train and test splits and identified the p-threshold that best generalized within the discovery

480 dataset (range of $p=0.001$ to $p=0.1$, p-value selected based on the best predictive performance,

481 see Supplementary Fig. 3, 4). Finally, to generate a final model that can be tested in the hold-out

482 sample and is consistent with other studies(62,65), we only kept features that appear on all of the

483 CV folds; these were singled-out and refitted back to the whole discovery dataset (Fig. 1E). The

484 resulting linear regression model was then used to generate predictions in the hold-out sample to

485 assess generalizability (Fig. 1F). Performance metrics reported here are the correlation between

486 predicted pain scores by the model and actual pain scores reported by the subject $\left(r_{\text {predvsactual }}\right)$.

487 Functional and structural connectivity were modelled separately. As an additional quality control

488 step, within the discovery and hold-out datasets, we exclude outliers - participants whose mean

489 connectivity values are more than two standard deviations away from the group mean

490 connectivity(66). This led to an exclusion of four participants from the rsfMRI dataset (all from

491 the discovery dataset) and four participants from the DTI dataset (two from the discovery dataset,

492 two from the hold-out dataset).

493

494 Assessing generalizability of the machine-learning results

495 The above method can also be applied to less constrained models. To test the generalizability of

496 the machine-learning pipeline under less stringent conditions, we tested the predictive ability of a 
simpler model by also performing univariate feature selection but instead feeding the selected features directly into a linear regression (i.e. not separating positive and negative correlations, and not summing all features into a single value). Again, in each CV-fold, significant features are selected and the threshold is determined by selecting the best performing model using the CVsamples. Models were validated like above. The same discovery and hold-out samples were used.

\section{Acknowledgements}

504 The authors would like to thank all members of the Apkarian lab for their feedback on the 505 manuscript. This work was supported by the Office of the Assistant Secretary of Defense for 506 Health Affairs under award W81XWH-15-1-0603. Opinions, interpretations, conclusions, and 507 recommendations are those of the authors and are not necessarily endorsed by the Department of 508 Defense. This work was further supported by National Institutes of Health grant P50 DA044121 509 and grant R01AR074274-01A1.

\section{References}

1. Thurman DJ, Alverson C, Dunn KA, Guerrero J, Sniezek JE. Traumatic Brain Injury in the United States: A Public Health Perspective: Journal of Head Trauma Rehabilitation. 1999 Dec;14(6):602-15.

2. Hartvigsen J, Boyle E, Cassidy JD, Carroll U. Mild Traumatic Brain Injury After Motor Vehicle Collisions: What Are the Symptoms and Who Treats Them? A Population-Based 1-Year Inception Cohort Study. Archives of Physical Medicine and Rehabilitation. 2014 Mar;95(3):S286-94.

3. Sterling M, Jull G, Kenardy J. Physical and psychological factors maintain long-term

4. Khokhar BR, Lindberg MA, Walker WC. Post-mTBI Pain Interference in a U.S. Military Feb 26;186(3-4):e293-9. 
5. Pettersson K, Hildingsson C, Toolanen G, Fagerlund M, Björnebrink J. MRI and neurology in acute whiplash trauma: No correlation in prospective examination of 39 cases. Acta Orthopaedica Scandinavica. 1994 Jan;65(5):525-8.

6. Anderson SE, Boesch C, Zimmermann H, Busato A, Hodler J, Bingisser R, et al. Are There Cervical Spine Findings at MR Imaging That Are Specific to Acute Symptomatic Whiplash Injury? A Prospective Controlled Study with Four Experienced Blinded Readers. Radiology. 2012 Feb;262(2):567-75.

7. Ronnen HR, de Korte PJ, Brink PR, van der Bijl HJ, Tonino AJ, Franke CL. Acute whiplash injury: is there a role for MR imaging?--a prospective study of 100 patients. Radiology. 1996 Oct;201(1):93-6.

8. Kuperman P, Granovsky Y, Granot M, Bahouth H, Fadel S, Hyams G, et al. Psychophysicpsychological dichotomy in very early acute mTBI pain: A prospective study. Neurology. 2018 Sep 4;91(10):e931-8.

9. Kuperman P, Granovsky Y, Bahouth H, Fadel S, Ben Lulu H, Bosak N, et al. Explaining very early acute mild traumatic brain injury after motor vehicle collision pain variability: additive value of pain sensitivity questionnaire. PR9. 2020 May;5(3):e821.

10. Sarrami P, Armstrong E, Naylor JM, Harris IA. Factors predicting outcome in whiplash injury: a systematic meta-review of prognostic factors. J Orthopaed Traumatol. 2017 Mar;18(1):9-16.

11. Van Oosterwijck J, Nijs J, Meeus M, Paul L. Evidence for central sensitization in chronic whiplash: A systematic literature review: Systematic review: Central sensitization in chronic WAD. EJP. 2013 Mar;17(3):299-312.

12. Saadon-Grosman N, Arzy S, Loewenstein Y. Hierarchical cortical gradients in somatosensory processing. Neurolmage. 2020 Nov;222:117257.

13. Behrens TEJ, Johansen-Berg H, Woolrich MW, Smith SM, Wheeler-Kingshott CAM, Boulby PA, et al. Non-invasive mapping of connections between human thalamus and cortex using diffusion imaging. Nat Neurosci. 2003 Jul;6(7):750-7.

14. Grömping U. Relative Importance for Linear Regression in $R$ : The Package relaimpo. J Stat Soft [Internet]. 2006 [cited 2021 Jul 20];17(1). Available from: http://www.jstatsoft.org/v17/i01/

15. Wager TD, Atlas LY, Lindquist MA, Roy M, Woo C-W, Kross E. An fMRI-Based Neurologic Signature of Physical Pain. N Engl J Med. 2013 Apr 11;368(15):1388-97.

16. Lee J-J, Kim HJ, Čeko M, Park B, Lee SA, Park H, et al. A neuroimaging biomarker for sustained experimental and clinical pain. Nat Med. 2021 Jan;27(1):174-82. 
17. Spisak T, Kincses B, Schlitt F, Zunhammer M, Schmidt-Wilcke T, Kincses ZT, et al. Pain-free resting-state functional brain connectivity predicts individual pain sensitivity. Nat Commun. 2020 Dec;11(1):187.

18. Sterling M, Jull G, Vicenzino B, Kenardy J. Characterization of acute whiplash-associated disorders. Spine (Phila Pa 1976). 2004 Jan 15;29(2):182-8.

19. Leth-Petersen S, Rotger GP. Long-term labour-market performance of whiplash claimants. J Health Econ. 2009 Sep;28(5):996-1011.

20. Jensen KB, Regenbogen C, Ohse MC, Frasnelli J, Freiherr J, Lundström JN. Brain activations during pain: a neuroimaging meta-analysis of patients with pain and healthy controls. Pain. 2016 Jun;157(6):1279-86.

21. Petrof I, Viaene AN, Sherman SM. Properties of the primary somatosensory cortex projection to the primary motor cortex in the mouse. Journal of Neurophysiology. 2015 Apr;113(7):2400-7.

22. Umeda $T$, Isa $T$, Nishimura $Y$. The somatosensory cortex receives information about motor output. Sci Adv. 2019 Jul;5(7):eaaw5388.

23. Lin LD, Murray GM, Sessle BJ. The effect of bilateral cold block of the primate face primary somatosensory cortex on the performance of trained tongue-protrusion task and biting tasks. Journal of Neurophysiology. 1993 Sep 1;70(3):985-96.

24. Brochier T, Boudreau M-J, Paré M, Smith AM. The effects of muscimol inactivation of small regions of motor and somatosensory cortex on independent finger movements and force control in the precision grip. Experimental Brain Research. 1999 Sep 3;128(1-2):31-40.

25. Pavlides C, Miyashita E, Asanuma H. Projection from the sensory to the motor cortex is important in learning motor skills in the monkey. Journal of Neurophysiology. 1993 Aug $1 ; 70(2): 733-41$.

26. Hiraba, Yayoi Yamaguchi, Hironori S H. Deficits of masticatory movements caused by lesions in the orofacial somatosensory cortex of the awake cat. Somatosensory \& Motor Research. 2000 Jan;17(4):361-72.

27. Angel RW, Malenka RC. Velocity-dependent suppression of cutaneous sensitivity during movement. Experimental Neurology. 1982 Aug;77(2):266-74.

28. Jiang W, Chapman CE, Lamarre Y. Modulation of somatosensory evoked responses in the primary somatosensory cortex produced by intracortical microstimulation of the motor cortex in the monkey. Exp Brain Res [Internet]. 1990 Apr [cited 2021 Jul 20];80(2). Available from: http://link.springer.com/10.1007/BF00228160 
29. Cui F, Arnstein D, Thomas RM, Maurits NM, Keysers C, Gazzola V. Functional Magnetic Resonance Imaging Connectivity Analyses Reveal Efference-Copy to Primary Somatosensory Area, BA2. Liu Y, editor. PLoS ONE. 2014 Jan 8;9(1):e84367.

30. Biswal B, Zerrin Yetkin F, Haughton VM, Hyde JS. Functional connectivity in the motor cortex of resting human brain using echo-planar mri. Magn Reson Med. 1995 Oct;34(4):537-41.

31. Holmes SA, Kim A, Borsook D. The brain and behavioral correlates of motor-related analgesia (MRA). Neurobiology of Disease. 2021 Jan;148:105158.

32. Emerson NM, Zeidan F, Lobanov OV, Hadsel MS, Martucci KT, Quevedo AS, et al. Pain Sensitivity is Inversely Related to Regional Grey Matter Density in the Brain. Pain. 2014 Mar;155(3):566-73.

33. Erpelding N, Moayedi M, Davis KD. Cortical thickness correlates of pain and temperature sensitivity. Pain. 2012 Aug;153(8):1602-9.

34. Liu Y, Latremoliere A, Li X, Zhang Z, Chen M, Wang X, et al. Touch and tactile neuropathic pain sensitivity are set by corticospinal projections. Nature. 2018 Sep;561(7724):547-50.

35. Duhamel J-R, Colby CL, Goldberg ME. Ventral Intraparietal Area of the Macaque: Congruent Visual and Somatic Response Properties. Journal of Neurophysiology. 1998 Jan 1;79(1):126-36.

36. Lobanov OV, Quevedo AS, Hadsel MS, Kraft RA, Coghill RC. Frontoparietal mechanisms supporting attention to location and intensity of painful stimuli. Pain. 2013 Sep;154(9):1758-68.

37. Yeterian EH, Pandya DN. Corticothalamic connections of the posterior parietal cortex in the rhesus monkey. J Comp Neurol. 1985 Jul 15;237(3):408-26.

38. Tu Y, Fu Z, Mao C, Falahpour M, Gollub RL, Park J, et al. Distinct thalamocortical network dynamics are associated with the pathophysiology of chronic low back pain. Nat Commun. 2020 Dec;11(1):3948.

39. Baliki MN, Geha PY, Apkarian AV, Chialvo DR. Beyond Feeling: Chronic Pain Hurts the Brain, Disrupting the Default-Mode Network Dynamics. Journal of Neuroscience. 2008 Feb 6;28(6):1398-403.

40. Belin P, Zatorre RJ, Ahad P. Human temporal-lobe response to vocal sounds. Brain Res Cogn Brain Res. 2002 Feb;13(1):17-26.

41. Assaf Y, Pasternak O. Diffusion Tensor Imaging (DTI)-based White Matter Mapping in Brain Research: A Review. J Mol Neurosci. 2008 Jan 1;34(1):51-61. 
42. Jones DK, Cercignani M. Twenty-five pitfalls in the analysis of diffusion MRI data. NMR Biomed. 2010 Aug;23(7):803-20.

43. Palacios EM, Owen JP, Yuh EL, Wang MB, Vassar MJ, Ferguson AR, et al. The evolution of white matter microstructural changes after mild traumatic brain injury: A longitudinal DTI and NODDI study. Sci Adv. 2020 Aug;6(32):eaaz6892.

44. Yuh EL, Cooper SR, Mukherjee P, Yue JK, Lingsma HF, Gordon WA, et al. Diffusion tensor imaging for outcome prediction in mild traumatic brain injury: a TRACK-TBI study. J Neurotrauma. 2014 Sep 1;31(17):1457-77.

45. Genc S, Anderson V, Ryan NP, Malpas CB, Catroppa C, Beauchamp MH, et al. Recovery of White Matter following Pediatric Traumatic Brain Injury Depends on Injury Severity. Journal of Neurotrauma. 2017 Feb 15;34(4):798-806.

46. Tavor I, Botvinik-Nezer R, Bernstein-Eliav M, Tsarfaty G, Assaf Y. Short-term plasticity following motor sequence learning revealed by diffusion magnetic resonance imaging. Human Brain Mapping. 2020;41(2):442-52.

47. Hofstetter S, Tavor I, Moryosef ST, Assaf Y. Short-Term Learning Induces White Matter Plasticity in the Fornix. J Neurosci. $2013 \mathrm{Jul}$ 31;33(31):12844-50.

48. Greicius MD, Supekar K, Menon V, Dougherty RF. Resting-State Functional Connectivity Reflects Structural Connectivity in the Default Mode Network. Cereb Cortex. 2009 Jan;19(1):72-8.

49. Honey CJ, Sporns O, Cammoun L, Gigandet X, Thiran JP, Meuli R, et al. Predicting human resting-state functional connectivity from structural connectivity. PNAS. 2009 Feb 10;106(6):2035-40.

50. Gil C, Decq P. How similar are whiplash and mild traumatic brain injury? A systematic review. Neurochirurgie. 2021 May;67(3):238-43.

51. Zigmond AS, Snaith RP. The hospital anxiety and depression scale. Acta Psychiatr Scand. 1983 Jun;67(6):361-70.

52. Sullivan MJL, Bishop SR, Pivik J. The Pain Catastrophizing Scale: Development and validation. Psychological Assessment. 1995;7(4):524-32.

53. Ruscheweyh R, Marziniak M, Stumpenhorst F, Reinholz J, Knecht S. Pain sensitivity can be assessed by self-rating: Development and validation of the Pain Sensitivity Questionnaire. Pain. 2009 Nov;146(1-2):65-74.

54. Cohen S, Kamarck T, Mermelstein R. A global measure of perceived stress. Journal of Health and Social Behavior. 1983;24(4):385-96. 
55. Jenkinson M, Beckmann CF, Behrens TEJ, Woolrich MW, Smith SM. FSL. Neurolmage. 2012 Aug 15;62(2):782-90.

56. Smith SM. Fast robust automated brain extraction. Hum Brain Mapp. 2002 Nov;17(3):14355.

57. Zhang Y, Brady M, Smith S. Segmentation of brain MR images through a hidden Markov random field model and the expectation-maximization algorithm. IEEE Trans Med Imaging. 2001 Jan;20(1):45-57.

58. Jenkinson M, Bannister $\mathrm{P}$, Brady $\mathrm{M}$, Smith S. Improved optimization for the robust and accurate linear registration and motion correction of brain images. Neuroimage. 2002 Oct;17(2):825-41.

59. Andersson JLR, Sotiropoulos SN. An integrated approach to correction for off-resonance effects and subject movement in diffusion MR imaging. Neurolmage. 2016 Jan;125:106378.

60. Behrens TEJ, Berg HJ, Jbabdi S, Rushworth MFS, Woolrich MW. Probabilistic diffusion tractography with multiple fibre orientations: What can we gain? Neuroimage. 2007 Jan 1;34(1):144-55.

61. Bastiani M, Cottaar M, Fitzgibbon SP, Suri S, Alfaro-Almagro F, Sotiropoulos SN, et al. Automated quality control for within and between studies diffusion MRI data using a nonparametric framework for movement and distortion correction. Neurolmage. 2019 Jan;184:801-12.

62. Shen X, Finn ES, Scheinost D, Rosenberg MD, Chun MM, Papademetris X, et al. Using connectome-based predictive modeling to predict individual behavior from brain connectivity. Nat Protoc. 2017 Mar;12(3):506-18.

63. Finn ES, Shen X, Scheinost D, Rosenberg MD, Huang J, Chun MM, et al. Functional connectome fingerprinting: identifying individuals using patterns of brain connectivity. Nat Neurosci. 2015 Nov;18(11):1664-71.

64. Kennard RW, Stone LA. Computer Aided Design of Experiments. Technometrics. 1969 Feb;11(1):137-48.

65. Rosenberg MD, Finn ES, Scheinost D, Papademetris X, Shen X, Constable RT, et al. A neuromarker of sustained attention from whole-brain functional connectivity. Nat Neurosci. 2016 Jan;19(1):165-71. connectivity uncovers the influence of participant's motion in functional magnetic resonance imaging studies. Human Brain Mapping. 2021;42(3):713-23. 


\section{Competing interests}

693 The authors declare that the research was conducted in the absence of any commercial or financial

694 relationships that could be construed as a potential conflict of interest

\section{Figure Captions} pain to obtain a linear equation predicting pain, which was tested in out-of-sample subjects. Lowerpanel, validation approach: the above approach was embedded into a machine-learning algorithm

D. The total sample here was separated into a

709 improve model stability, after the best $p$-value was determined, a new model was built based only

710 on the features that were significant in all the CV folds (features occurring in 100\% of the 10 -fold

711 CV iterations). F. Model performance was assessed within-sample by applying the linear equation

712 to the discovery dataset and out-of-sample by applying the linear equation in the hold-out dataset

713 to assess generalization. 
716 pain. Three features were selected by the machine-learning algorithm (A), and a model was cast.

717 The model's predicted values were significantly correlated with actual pain in the discovery dataset

718 (B, left) and in the hold-out dataset (B, middle) with equivalent effect sizes. The model was also 719 able to predict pain in a third independent group of mTBI patients with no whiplash-like symptoms

720 (B, right). Meta-analytical predictive performance metrics were calculated to summarize the

721 results from the three groups $(\mathbf{C})$, showing again reliable associations between predicted and

722 observed pain. (D) Raw connectivity strength and their association with pain are illustrated,

723 showing a negative association whereby more connections lead to less reported pain. (E)

724 Connectivity strength of the three tracts compared between subjects with WAD scores of 0,1 , and

7252 show no significant differences, supporting no association between symptom severity and 726 connectivity strength. left superior parietal Lobule, L SPL; left superior lateral occipital cortex, 727 sLOC.

Figure 3. Somatotopic organization of structural connectivity within the sensorimotor cortex and the thalamocortical system reveal topographically appropriate linkages related to acute mTBI pain. Post-hoc analysis looking at left PreC-PostC and left Thal-SPL connectivity at a

732 higher granularity. (A) Within the sensorimotor homunculi, the most predictive connectivity is 733 between the face and arm ROIs. Connectivity between the precentral arm and postcentral trunk 734 (site of injury, i.e. head/neck representation in the motor and sensory homunculi, respectively) also 735 show significant correlation with observed pain. (B) For the thalamus, the strongest predictor was 736 the VPL nuclei (top panel), together with an anterior/medial region. The VPL nuclei was also the 737 nucleus with strongest absolute connectivity to the parietal cortex (bottom panel). ${ }^{*} p<.05 ;{ }^{* *} p$ 
$738<.01$; \# survives FDR correction for multiple comparisons. Thalamus ROIs can be depicted in the

739 bottom right panel.

741 Figure 4. A multi-parameter model for predicting acute and longer-term mTBI pain. (A)

742 Correlations between the predictive structural connectivity tracts and psychophysical-

743 psychological variables. Higher pressure-pain conditioned pain modulation (CPM) and

744 suprathreshold pain (Pain50) scores were associated with stronger connectivity, and lower pain

745 sensitivity questionnaire (PSQ) scores were associated with stronger connectivity. Individually,

746 left PreC-PostC was mostly associated with CPM, while R PP - sLOC was associated with

747 perceived stress scale (PSS). Mechanical (Mech. TS) and electrical (Elect. TS) temporal

748 summation, Heat-pain conditioned pain modulation (HP CPM), Pain Catastrophizing (PCS),

749 Anxiety and Depression did not show any significant associations. (B) Relative importance metrics

750 for connectivity, PSQ, CPM, and Pain50, when predicting pain. A model with the four parameters

751 (Brain, psychological, and psychophysical parameters) explains 31\% of the variance in pain, with

752 connectivity parameters accounting for $50 \%$ of the full model variance (i.e. $15 \%$ of unique variance

753 in observed pain).

755 Figure 5. Stability of structural connectivity related to acute mTBI pain over 12 months and

756 their relationship to long-term mTBI pain. (A). Model performance at predicting pain over time;

757 baseline parameters are associated with observed pain at baseline (BL), 1 month (1M), 3 months

758 (3M), 6 months (6M) and a year (1Y, marginally significant) after a motorized vehicle crash, but

759 not when controlling for baseline pain. (B, left panel) connectivity strength of the three predictive

760 features measured at 6 months post-MVC is still significantly associated with reported pain at 6 
bioRxiv preprint doi: https://doi.org/10.1101/2021.11.12.468345; this version posted November 13,2021 . The copyright holder for this preprint (which was not certified by peer review) is the author/funder, who has granted bioRxiv a license to display the preprint in perpetuity. It is made available under aCC-BY 4.0 International license.

761 months, but not at 12 months. (B, right panel) connectivity remains stable from baseline to 6

762 months, and after one year. Lines represent paired differences within subject. * $p<.05 ; * * p<.01$;

$763 * * * p<.001 ; \& p=.051$ 
A Correlate each edge with pain across subjects

Brain Pain

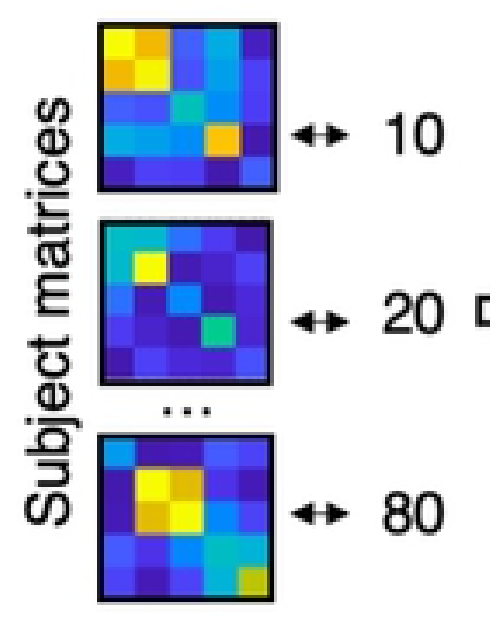

Sig. edges

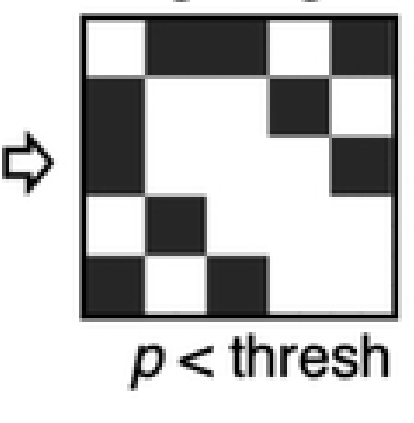

D Tune params. with 10-fold CV in discovery dataset

Training folds Test fold

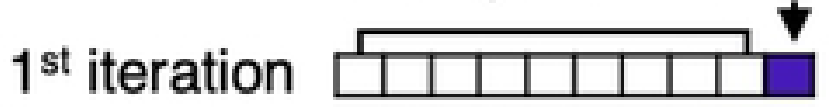

$2^{\text {nd }}$ iteration

10

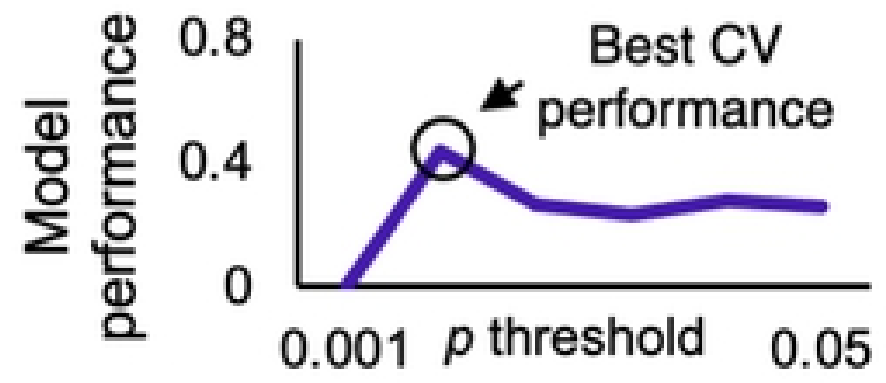

B Sum connectivity of significant edges

Subjects
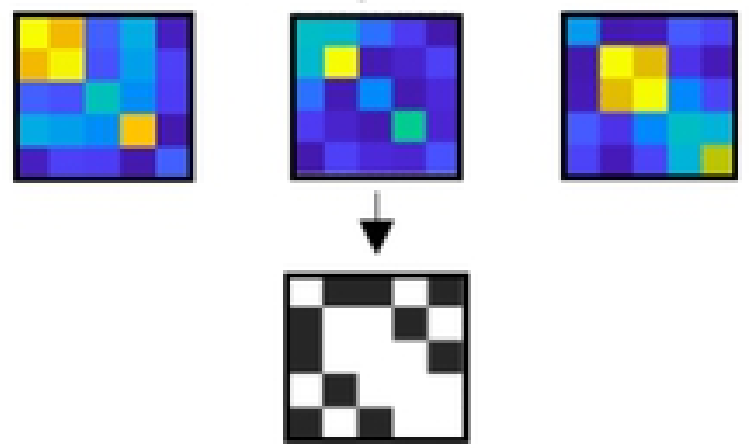

$\sum_{\mathrm{s} 1} \quad \sum_{\mathrm{s} 2} \quad \sum_{\mathrm{s} n}$

(brain parameters)

E Refit model with features selected in $100 \%$ of $\mathrm{CV}$ folds

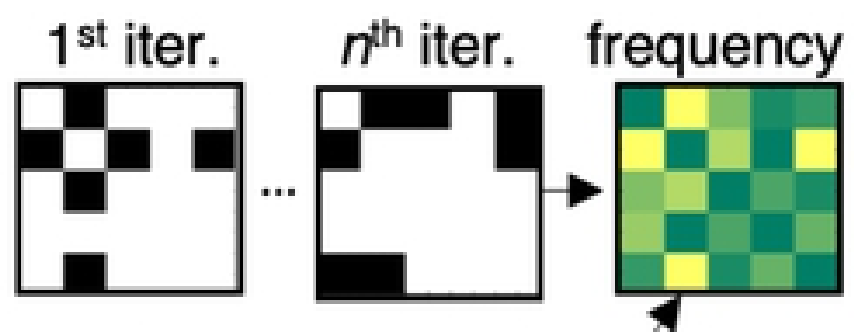

Features in all CV folds

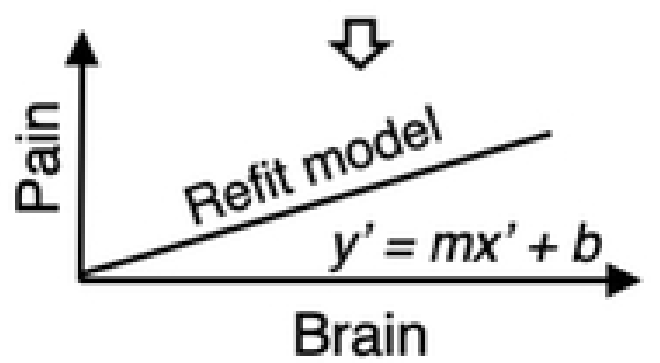

C Fit brain parameters to pain with a linear model

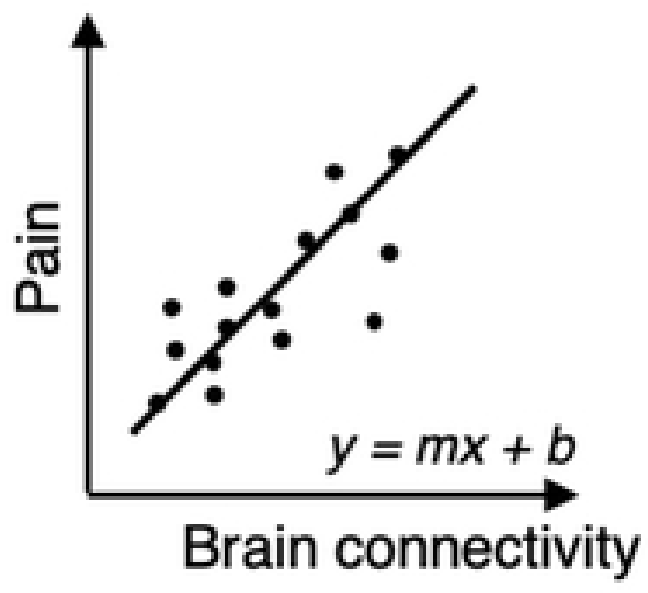

F Predict pain and assess model performance
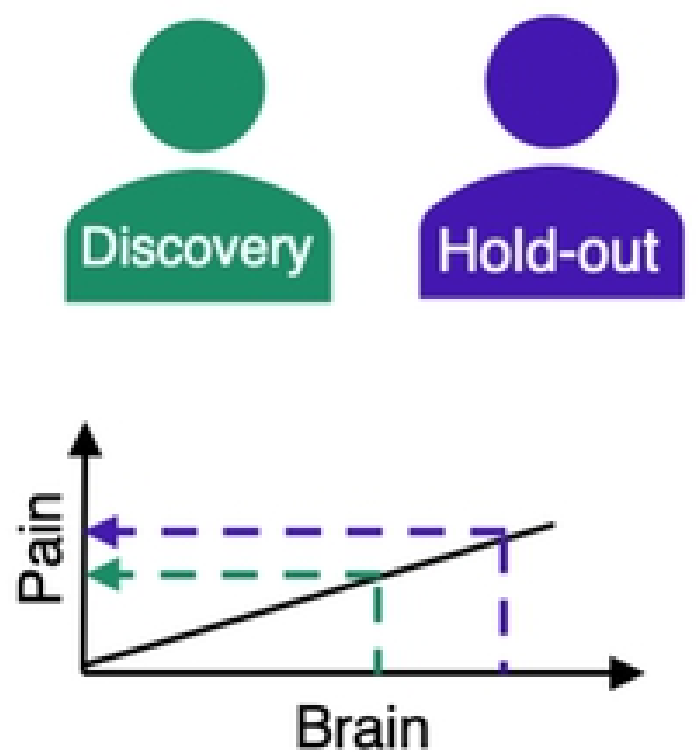
A

L Precentral to L Postcentral

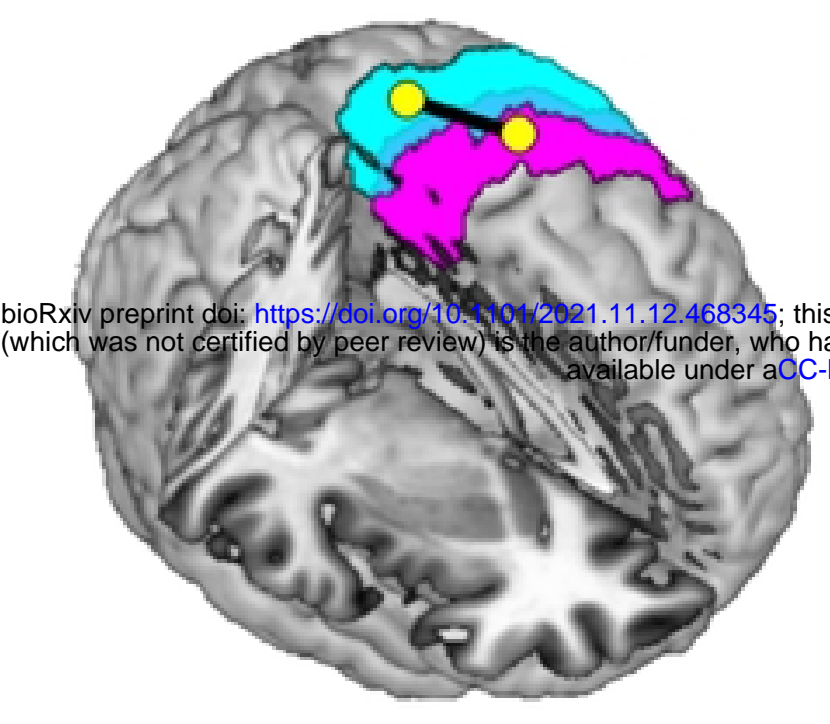

B

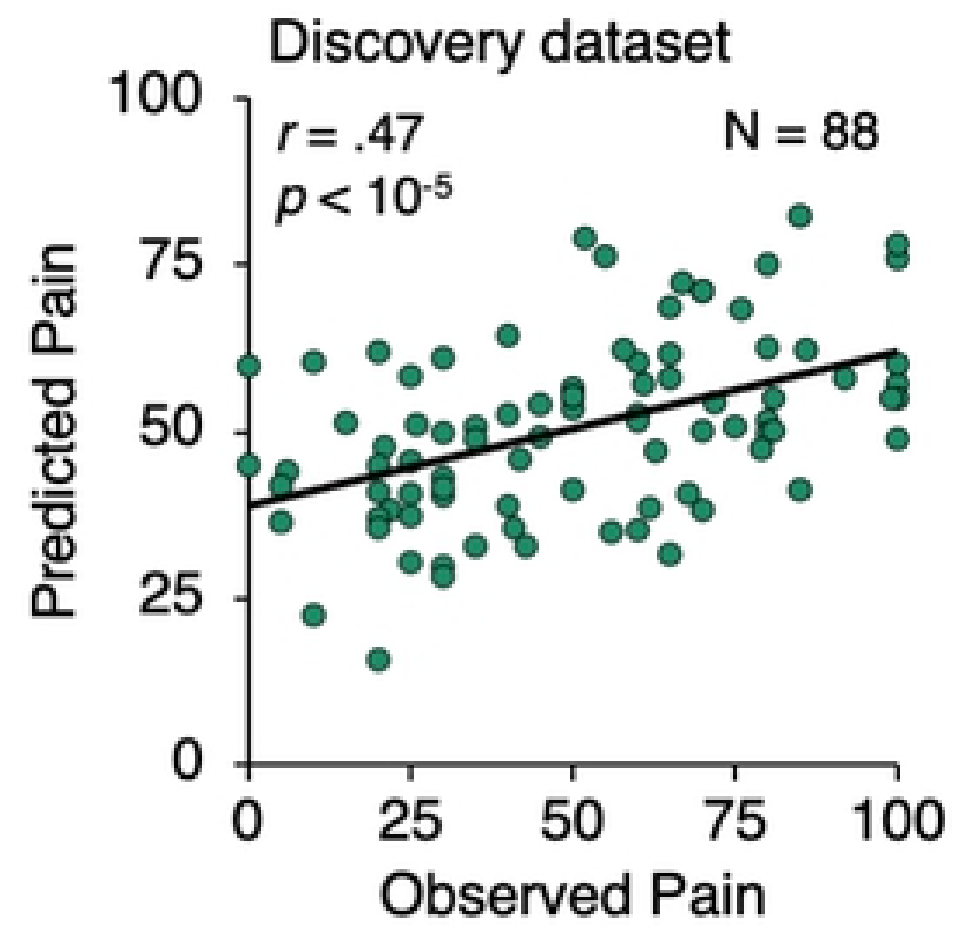

C

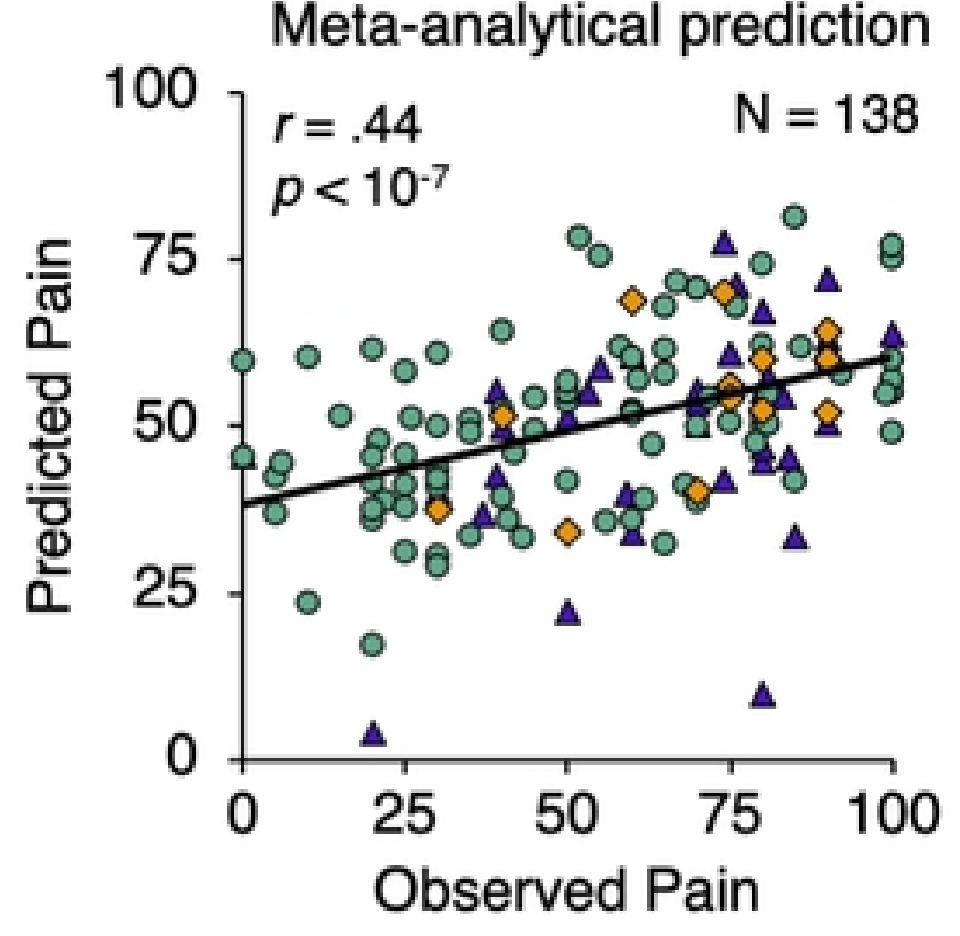

L SPL to L Thalamus
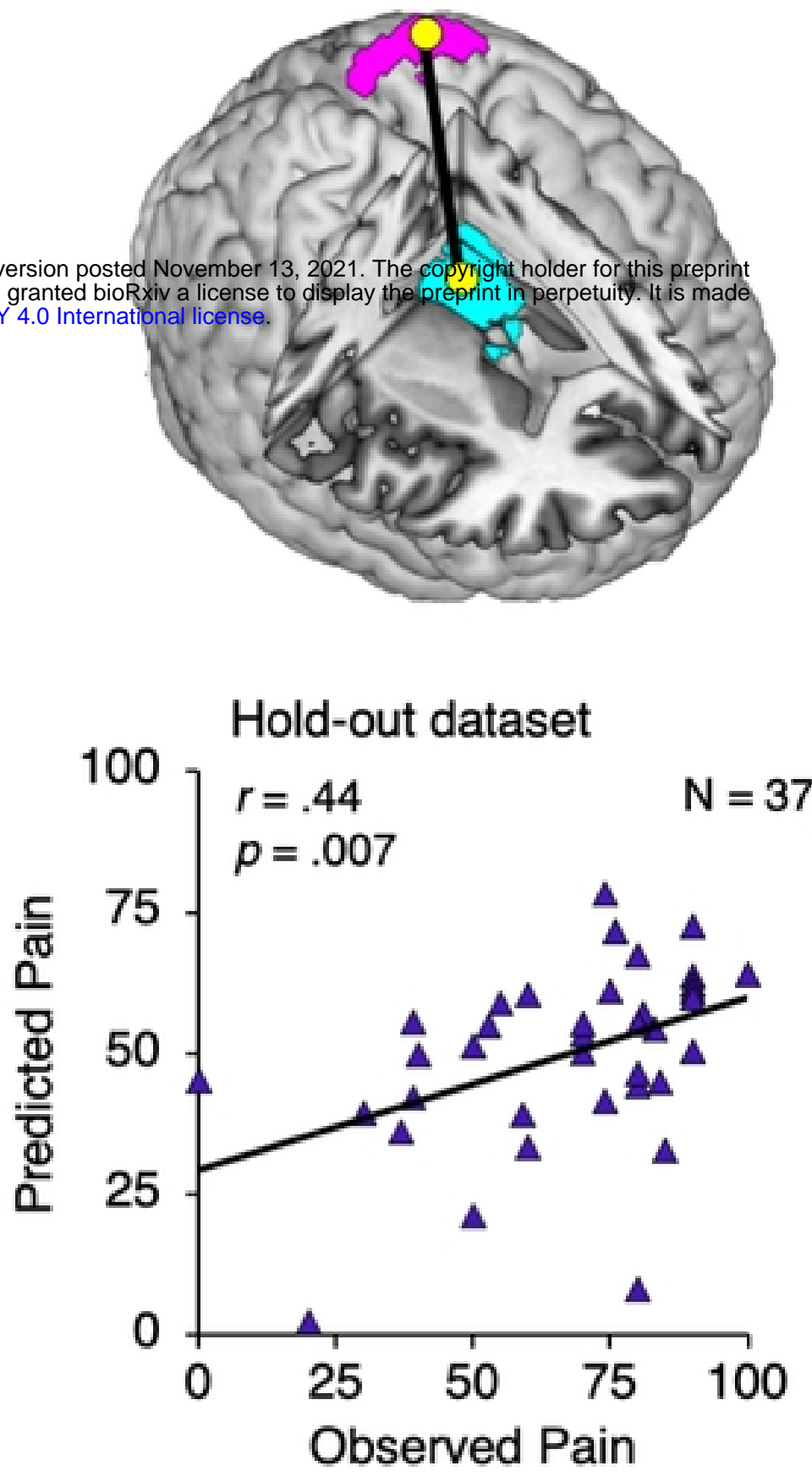

D

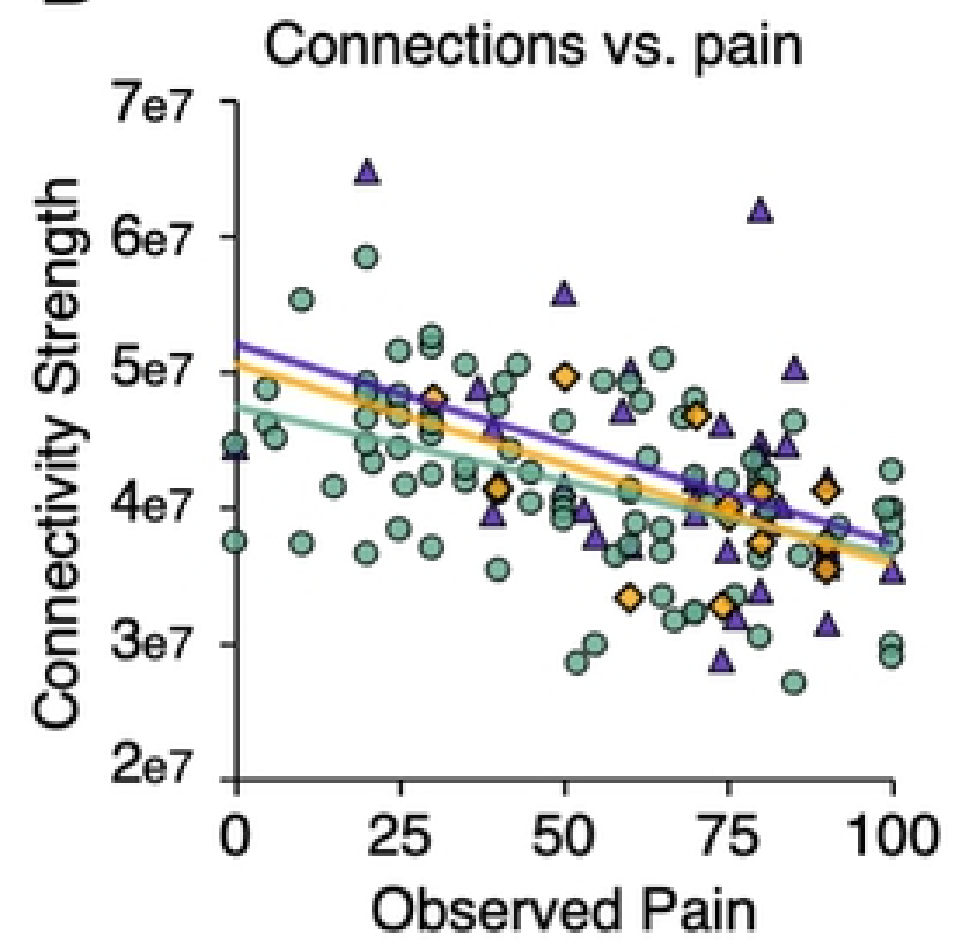

L sLOC to R Planum Polare
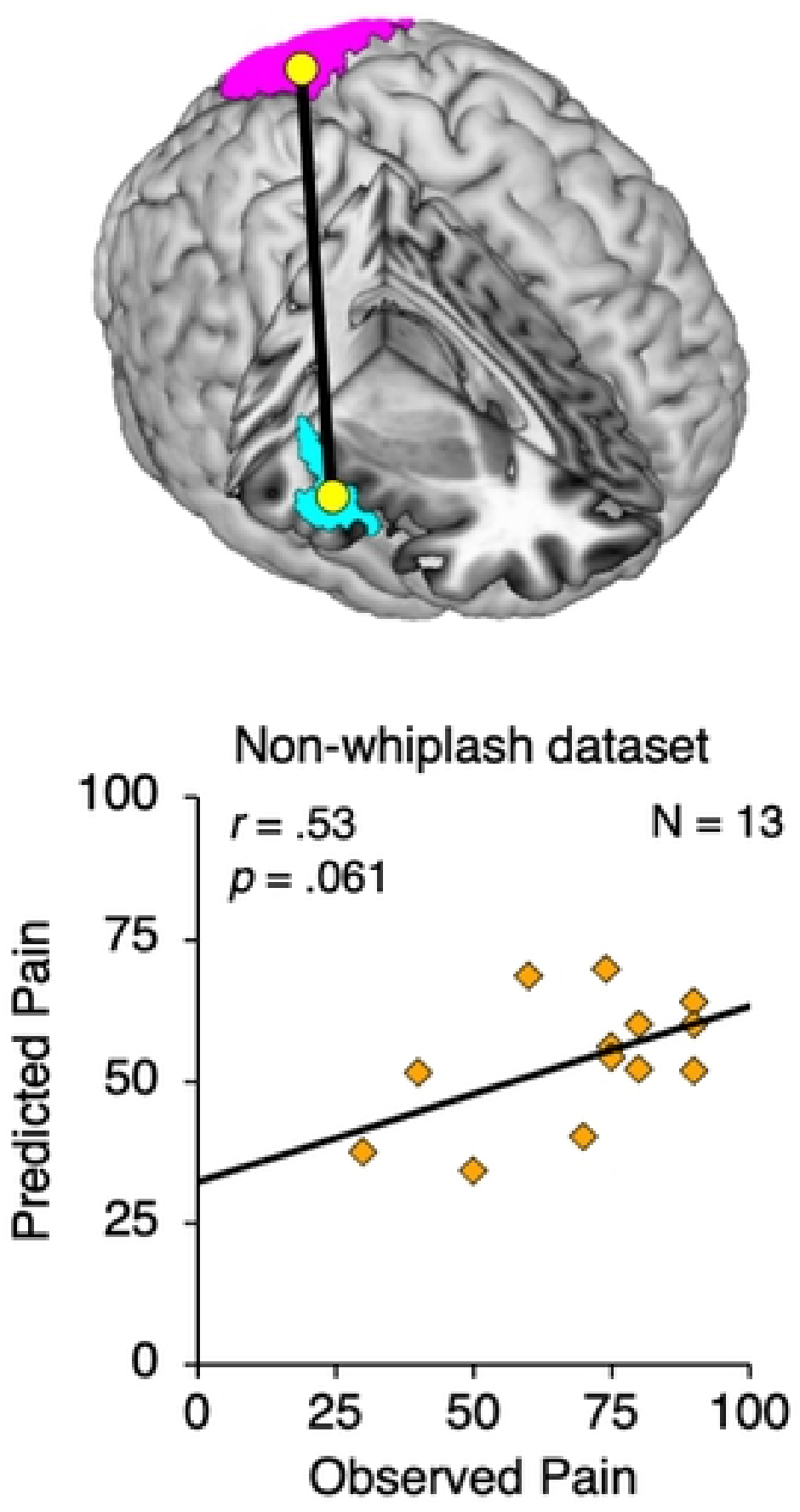

E

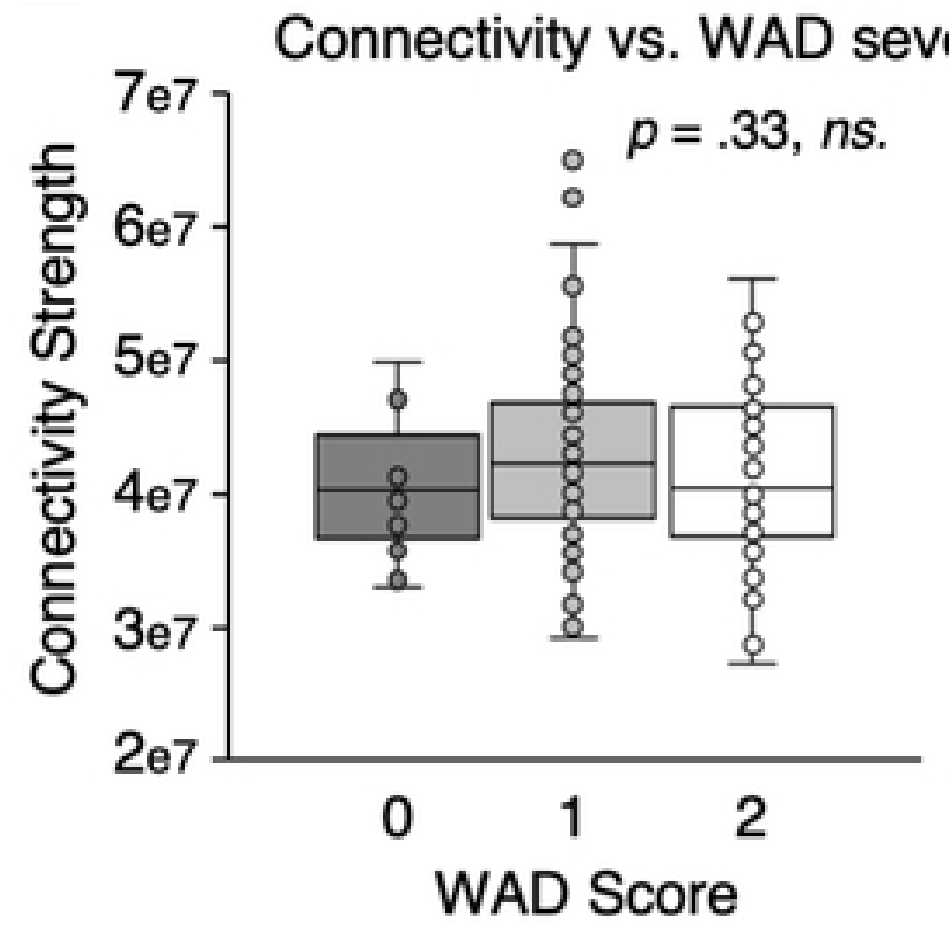

Discovery $\boldsymbol{\Delta}$ Hold-out $\diamond$ Non-whiplash 
A

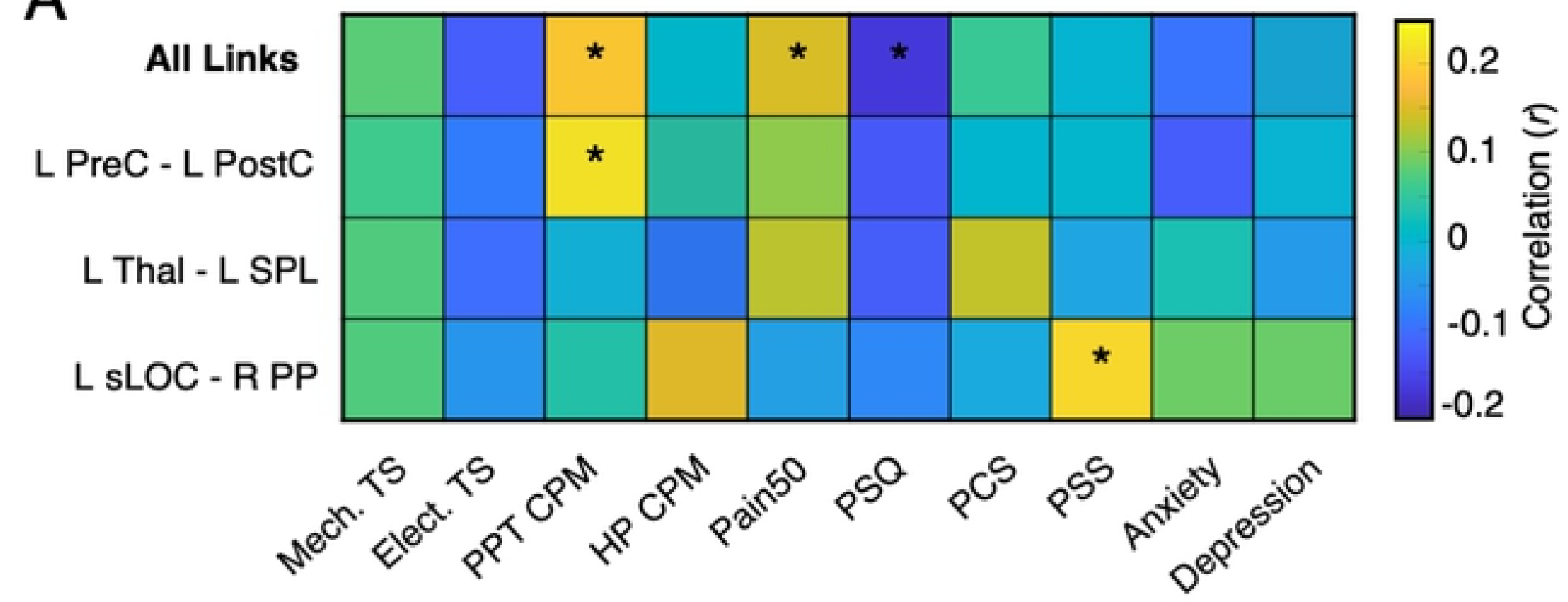

B $\quad 0.2\rceil^{\text {Total }} \mathrm{R}^{2}=31.28 \%$

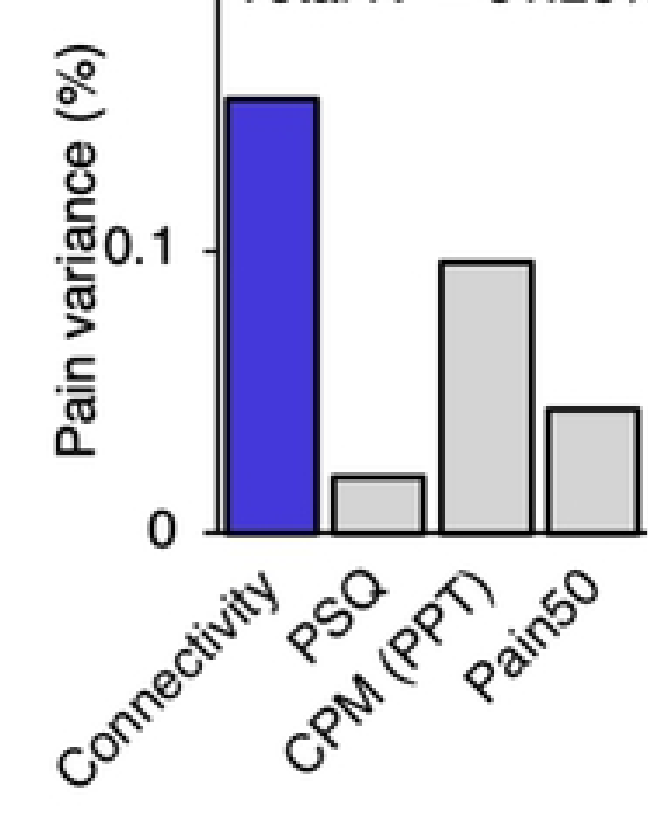


A

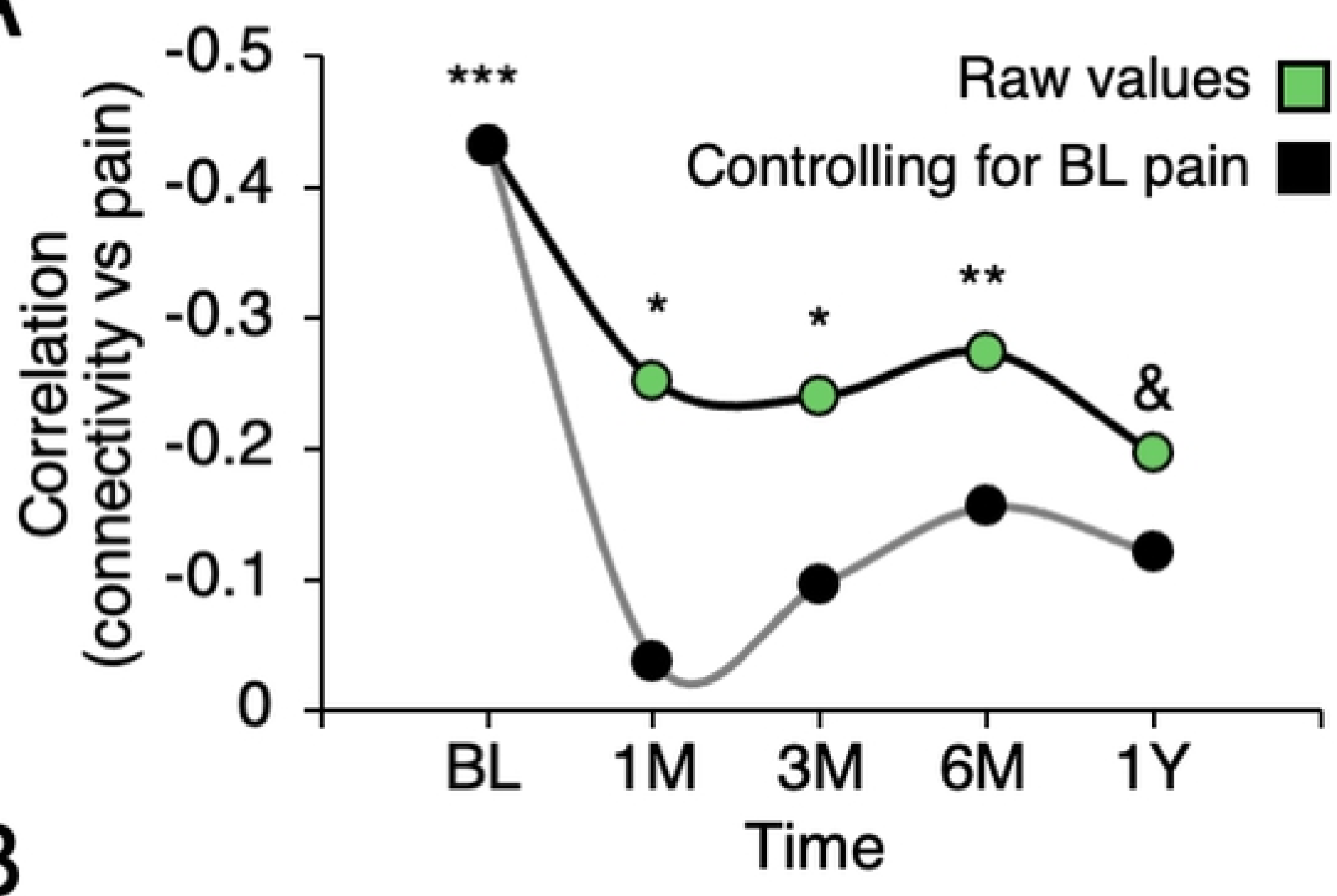

bioRxiv preprint doi: https://doi.org/10.1101/2021.11.12.468345; this version posted November 13,2021 . The copyright holder for this preprint
(which was not certified by peer review) is the author/funder, who has granted bioRxiv a license to display the preprint in perpetuity. It is made

6 months after injury $\quad$ Baseline vs. 6 months

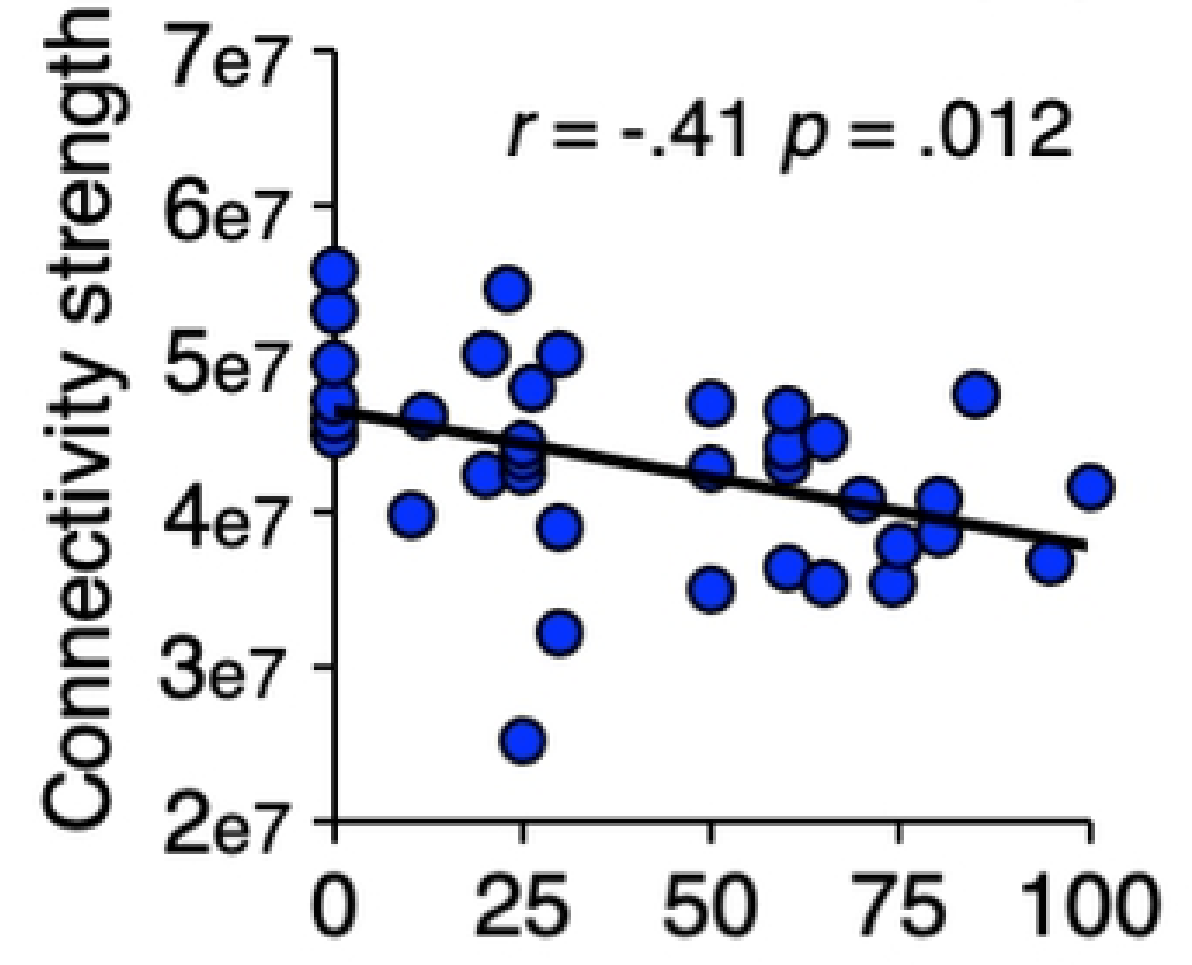

Observed pain (6M)

12 months after injury

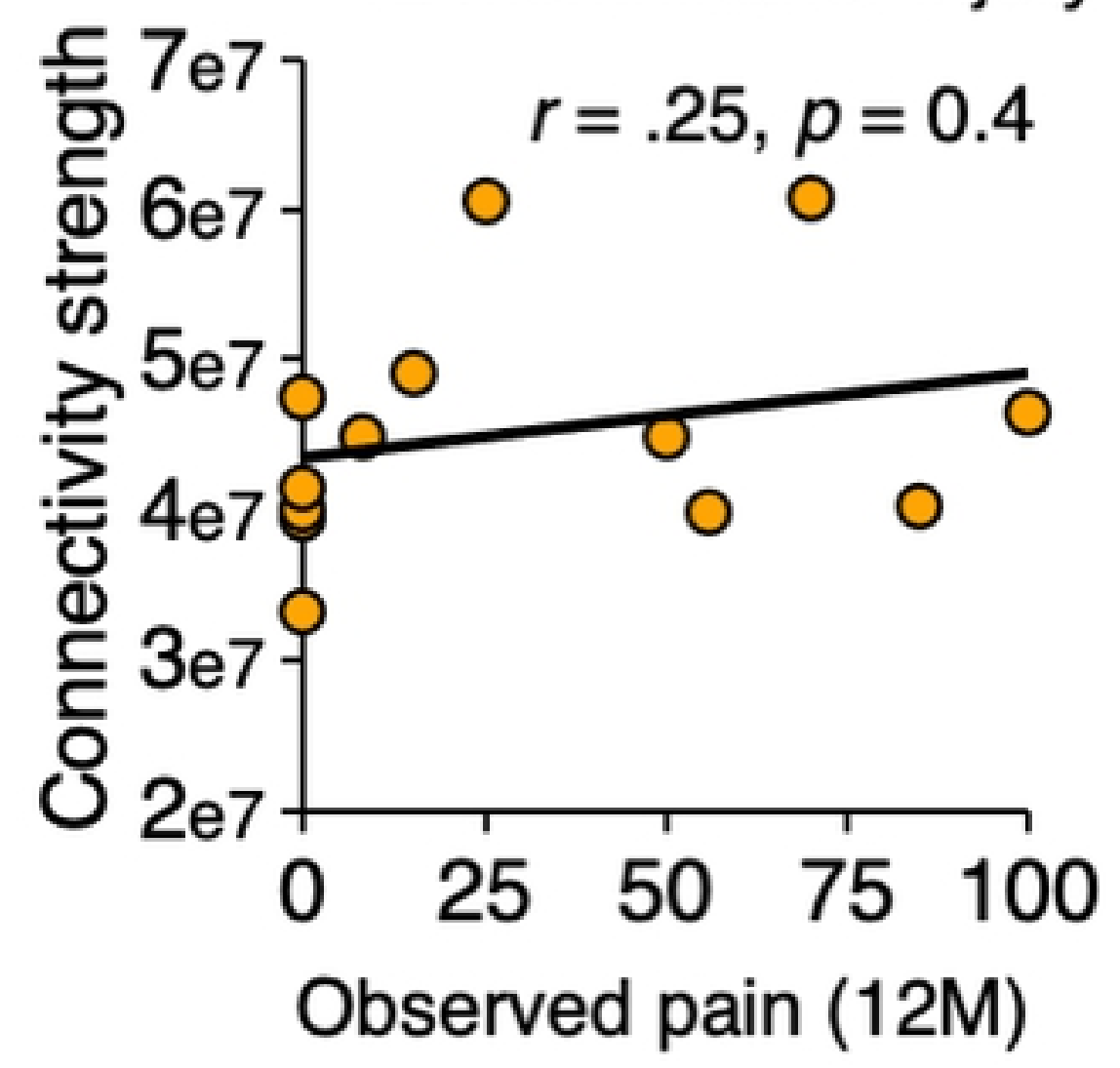

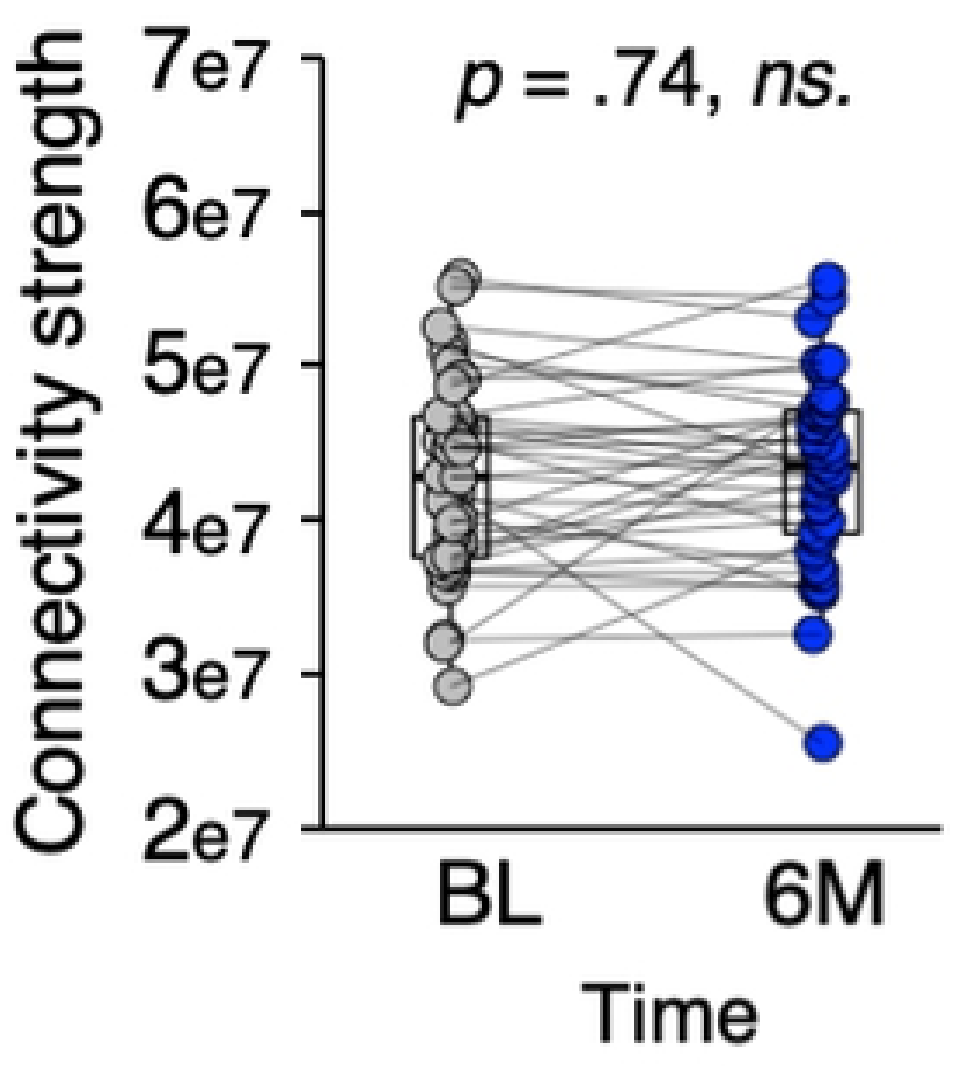

Baseline vs. 12 months

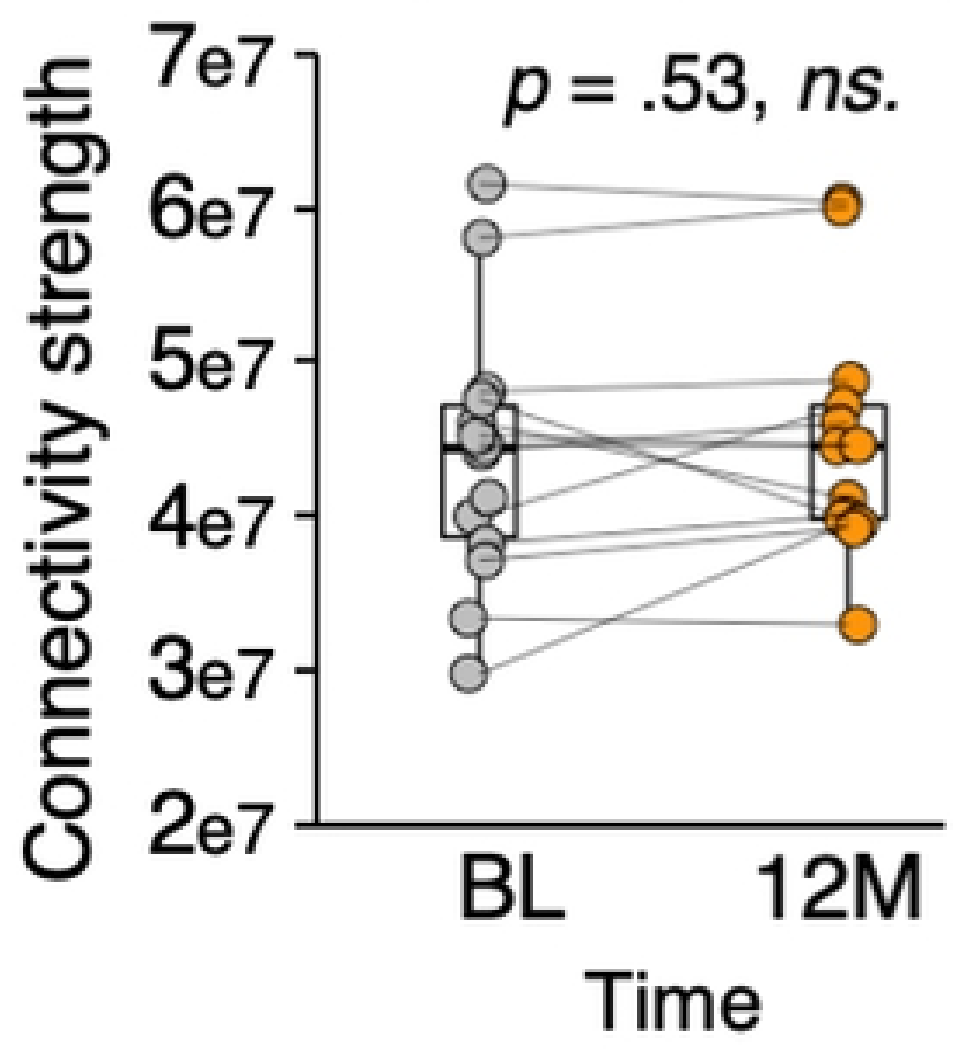

